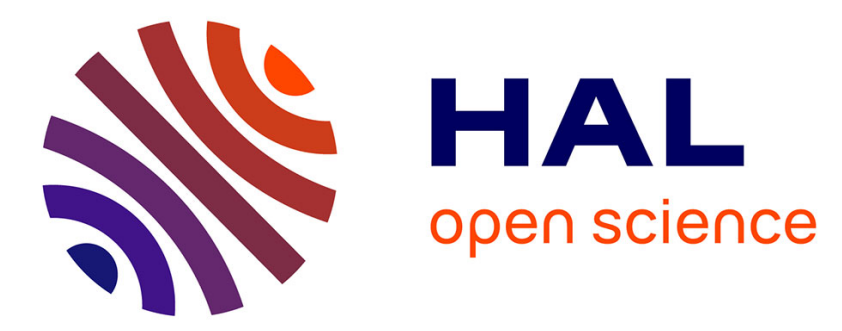

\title{
On the real zeros of positive semi-definite biquadratic forms
}

Ronan Quarez

\section{To cite this version:}

Ronan Quarez. On the real zeros of positive semi-definite biquadratic forms. Communications in Algebra, 2015, 43 (3), pp.1317-1353. 10.1080/00927872.2013.865052 . hal-00523238v2

\section{HAL Id: hal-00523238 \\ https://hal.science/hal-00523238v2}

Submitted on 7 Dec 2010

HAL is a multi-disciplinary open access archive for the deposit and dissemination of scientific research documents, whether they are published or not. The documents may come from teaching and research institutions in France or abroad, or from public or private research centers.
L'archive ouverte pluridisciplinaire HAL, est destinée au dépôt et à la diffusion de documents scientifiques de niveau recherche, publiés ou non, émanant des établissements d'enseignement et de recherche français ou étrangers, des laboratoires publics ou privés. 


\title{
ON THE REAL ZEROS OF POSITIVE SEMI-DEFINITE BIQUADRATIC FORMS
}

\author{
RONAN QUAREZ
}

\begin{abstract}
For a positive semi-definite biquadratic forms $F$ in $(3,3)$ variables, we prove that if $F$ has a finite number but at least 7 real zeros $\mathcal{Z}(F)$, then it is not a sum of squares. We show also that if $F$ has at least 11 zeros, then it has infinitely many real zeros and it is a sum of squares. It can be seen as the counterpart for biquadratic forms as the results of Choi, Lam and Reznick for positive semi-definite ternary sextics.

We introduce and compute some of the numbers $\mathrm{BB}_{n, m}$ which are set to be equal to $\sup |\mathcal{Z}(F)|$ where $F$ ranges over all the positive semi-definite biquadratic forms $F$ in $(n, m)$ variables such that $|\mathcal{Z}(F)|<\infty$.

We also recall some old constructions of positive semi-definite biquadratic forms which are not sums of squares and we give some new families of examples.
\end{abstract}

\section{INTRODUCTION}

Throughout this paper, a form will just denote an homogeneous polynomial over the reals and $\mathcal{H}_{n, k}$ the set of all forms of degree $k$ with $n$ variables. A form $F(x)$ with variables $\left(x_{0}, \ldots x_{n-1}\right)$ is said to be positive semi-definite if $F(x) \geq 0$ for all $x \in \mathbb{R}^{n}$. The form $F \in \mathcal{H}_{n, 2 k}$ is a sum of squares if it can be written $F(x)=\sum_{s=1}^{r} F_{s}^{2}(x)$ for some forms $F_{s} \in \mathcal{H}_{n, k}$.

The study of positive semi-definite forms which are not sums of squares has been (and still is) an intensive area of researchs since Hilbert showed (see Hil|) that the cone of positive semi-definite forms coincide with the cone of forms which are sum of squares if and only if $n=2$, or $k=2$, or $(n, k)=(3,4)$.

In CLR1, Choi, Lam and Reznick gave some more precise statement for ternary sextics and quaternary quartics, using the real zeros set of the forms viewed in some projective space. For instance, one of their main results is :

Theorem CLR1. If $f \in \mathcal{H}_{3,6}$ is positive semi-definite with at least 11 real zeros in the projective plane, then $f$ has infinitely many real zeros and it is a sum of squares.

Our main motivation is to establish a counterpart for biquadratic forms.

A form $F(x ; s)$ is called biquadratic in the variables $x=\left(x_{0}, \ldots, x_{n-1}\right)$ and $s=\left(s_{0}, \ldots, s_{m-1}\right)$ if for each $x \in \mathbb{R}^{n}, F(x ; s)$ is a quadratic form in the variables $s$; and for each $s \in \mathbb{R}^{m}, F(x ; s)$ is a quadratic form in the variables $x$. The set of all biquadratic forms with respect to the variables $(x ; s)$ is denoted by $\mathcal{B} Q_{n, m}$. A biquadratic form $F(x ; s)$ is said to be positive semi-definite if $F(x ; s) \geq 0$ for all

Date: December 7, 2010.

2000 Mathematics Subject Classification. 14P99; $15 \mathrm{~A} 63$.

Key words and phrases. Biquadratic Forms; Positive semi-definite; Real zeros; Sums of squares. 
$(x, s) \in \mathbb{R}^{n+m}$. Finally, the set of all positive semi-definite biquadratic forms in $\mathcal{B} Q_{n, m}$ is denoted by $\mathcal{P} Q_{n, m}$.

Biquadratic forms naturally arise in several areas of pure and applied mathematics, for instance in control theory and also in theoretical physics.

Among all the bibliography about biquadratic forms, we will mention first the result of Calderon (see $[\mathrm{Ca}$ ) which says that any positive semi-definite biquadratic form in $\mathcal{B} Q_{n, 2}$ is a sum of squares. Later, Choi in [C1] gave an example of a positive semi-definite biquadratic form in $\mathcal{B} Q_{3,3}$ which is not a sum of squares. Maybe less known, Terpstra already in $[\mathrm{Te}$ had also given a geometric construction of positive semi-definite biquadratic forms in $\mathcal{B} Q_{3,3}$ which are not a sum of squares.

Throughout the paper, we view the real zeros set $\mathcal{Z}(F)$ of a biquadratic forms $F \in \mathcal{H}_{n, m}$ in the product of projective spaces $\mathbb{P}^{n-1} \times \mathbb{P}^{m-1}$.

In section 4 , we set the numbers $\mathrm{BB}_{n, m}$ to be equal to $\sup |\mathcal{Z}(F)|$ where $F$ ranges over all biquadratic forms in $\mathcal{P} Q_{n, m}$ with $|\mathcal{Z}(F)|<\infty$. This definition is analogous to the definition, for forms in $\mathcal{H}_{n, k}$, of the numbers $B_{n, k}$ in [CLR1]. We will give some properties of the $\mathrm{BB}_{n, m}$ and compute some values, for instance we show that $\mathrm{BB}_{n, 2}=n$ using the well known values of the degrees of Segre varieties. We also prove that $7 \leq \mathrm{BB}_{3,3} \leq 10$.

In Section Section 5 we show that if $F \in \mathcal{P} Q_{3,3}$ has a finite number but at least 7 real zeros, then it is not a sum of squares. We show also that if $F$ has at least 11 zeros then it has infinitely many real zeros and it is a sum of squares.

In Section [6, we recall Terpstra's construction $[\mathrm{Te}]$ and give some variations around the Choi example C1. We also give some new families of examples of positive semi-definite non sum of squares biquadratic forms.

In Section 7, we study the possible configurations in $\mathbb{P}^{n-1} \times \mathbb{P}^{m-1}$ of the zero sets of biquadratic forms in $\mathcal{P} Q_{n, m}$. Of particular interest are the maximal configurations which are defined to be the finite subsets $\Omega \subset \mathbb{P}^{n-1} \times \mathbb{P}^{m-1}$ such that if $F \in \mathcal{P} Q_{n, m}$ vanishes at $\Omega$ and also at another point in $\mathbb{P}^{n-1} \times \mathbb{P}^{m-1}$, then $\mathcal{Z}(F)$ is infinite. We give some characterisations of maximal configurations in the case $(n, m)=(2,2)$ and $(n, m)=(3,2)$. In the case $(n, m)=(3,3)$, we give some necessary conditions and we also give a complete worked example .

For a maximal configuration $\Omega$, we are interested to determine $\mathcal{P} Q(\Omega)$ the set of all possible biquadratic forms $F \in \mathcal{P} Q_{3,3}$ such that $\Omega \subset \mathcal{Z}(F)$. This seems to be a difficult task in general. Again, we give a complete worked example when $(n, m)=(3,3)$.

\section{Preliminaries}

The set of all real matrices of size $n \times m$ is denoted by $M_{n, m}(\mathbb{R})$ or alternatively by $\mathbb{R}^{n \times m}$. The symbol ${ }^{T}$ will denote the transposition.

To any biquadratic form $F(x ; s)$ with variables $x=\left(x_{0}, \ldots, x_{n-1}\right)$ and $s=$ $\left(s_{0}, \ldots, s_{m-1}\right)$ running over $\mathbb{R}$, one may canonically associate a matrix $M_{s}(F)$ whose entries are forms in $\mathcal{H}_{m, 2}$ with variables $s$ (resp. a matrix $M_{x}(F)$ whose entries are forms in $\mathcal{H}_{m, 2}$ with variables $x$ ) such that

$$
F(x ; s)=x M_{s}(F) x^{T}=s M_{x}(F) s^{T} .
$$


Of course, the biquadratic form $F(x, y)$ is positive semi-definite if and only if the matrix $M_{s}(F)$ (respectively $M_{x}(F)$ ) is positive semi-definite for all $s \in \mathbb{R}^{m}$ (respectively for all $x \in \mathbb{R}^{n}$ ).

One motivation for studying biquadratic forms is that they are related to positive linear maps (see [C2] for background).

2.1. Positive linear maps. A linear map $\Phi: M_{n}(\mathbb{R}) \rightarrow M_{m}(\mathbb{R})$ preserving symmetry is said to be positive if the matrices $\Phi(A)$ are positive semi-definite for all symmetric positive semi-definite matrices $A \in M_{n}(\mathbb{R})$.

The linear map $\Phi$ is entirely determined by the image of positive semi-definite matrices of rank 1 . Indeed, the matrix $x^{T} x$ has rank 1 and we have

$$
\Phi\left(x^{T} x\right)=\sum_{1 \leq i, j \leq n} A_{i j} x_{i} x_{j},
$$

where $A_{i j} \in \mathbb{R}^{m \times m}$. Then, we define

$$
\phi(x ; s)=s \Phi\left(x^{T} x\right) s^{T}=s\left(\sum_{1 \leq i, j \leq n} x_{i} A_{i j} x_{j}\right) s^{T} .
$$

Although the notion of complete positivity does not work as well as in the complex case, an easy computation shows the property :

Proposition 2.1. Let $\Phi$ be a linear map preserving symmetry. The map $\Phi$ is positive if and only if the biquadratic form $\phi$ is positive semi-definite. Moreover, the following properties are equivalent:

a) there exists some $V_{k} \in \mathbb{R}^{n \times m}$ for $k \in\{1 \ldots, r\}$ such that

$$
\Phi(M)=\sum_{k=1}^{r} V_{k}^{T} M V_{k}
$$

for all $M$,

b) the biquadratic form $\phi$ is a sum of squares.

Let us mention that the complex counterpart of theses notions, namely positive semi-definite non sum of squares bihermitian forms, are important in theoretical physics (see for instance [SZ]).

2.2. Zeros in projective spaces. Let $F(x ; s)$ be a biquadratic form where $x=$ $\left(x_{0}, \ldots, x_{n-1}\right)$ and $s=\left(s_{0}, \ldots, s_{m-1}\right)$. To any non trivial zero $A_{0} \in \mathbb{R}^{n} \times \mathbb{R}^{m}$ of $F$, we may canonically associate an element $P_{0} \in \mathbb{P}^{n-1} \times \mathbb{P}^{m-1}$. Throughout the paper, we are considering real projective spaces.

We denote respectively by $\Pi_{1}$ and $\Pi_{2}$ the two projections of $\mathbb{P}^{n-1} \times \mathbb{P}^{m-1}$ onto $\mathbb{P}^{n-1}$ and respectively $\mathbb{P}^{m-1}$.

We denote by $\mathcal{Z}(F)$ the set of all real zeros of $F$ in $\mathbb{P}^{n-1} \times \mathbb{P}^{m-1}$. Here again, we emphasise that all the considered zeros are real.

Proposition 2.2. Let $F(x ; s) \in \mathcal{P} Q_{n, m}$ be a positive semi-definite biquadratic form.

a) There exists $S_{0}$ such that $P_{0}=\left(X_{0}, S_{0}\right) \in \mathbb{P}^{n-1} \times \mathbb{P}^{m-1}$ is a real zero of $F$ if and only if $\operatorname{det}\left(M_{x}(F)\right)$ vanishes at $X_{0}$. 
b) If we view the coefficients of $F(x ; s)$ as variables, the condition $F\left(P_{0}\right)=0$ where $P_{0} \in \mathbb{P}^{n-1} \times \mathbb{P}^{m-1}$ gives a set of $n+m-1$ linearly independent equations.

Proof. a) If $P_{0}$ is a real zero of $F$, the vector $S_{0}$ is an isotropic vector for the quadratic form defined by $M=M_{x}(F)\left(X_{0}\right)$. Since it is positive semidefinite, it must be a singular matrix : $\operatorname{det}(M)=0$.

The converse is easy : if $M$ is singular, it admits an isotropic vector $S_{0}$.

b) If $F\left(P_{0}\right)=0$, then $P_{0}$ necessarily is a critical point for $F$ and all the partial derivatives vanishes at $P_{0}$. By the Euler identity for forms, the cancellation of all partial derivatives implies that $F\left(P_{0}\right)=0$.

We may be more explicit. First, to simplify, we may assume that $P_{0}=$ $(1,0, \ldots, 0 ; 1,0, \ldots, 0)$. Let us write also $F(x ; s)=\sum_{i, j ; k, l} a_{i, j ; k, l} x_{i} x_{j} s_{k} s_{l}$. The cancellation of all partial derivatives gives $a_{i, 1 ; 1,1}=a_{1,1 ; k, 1}$ for all $i$ and $k$. We find a set of $n+m-1$ linearly independent equations.

\section{Biquadratic Forms in Dimensions $(n, 2)$}

3.1. The result of Calderon. The case of biquadratic forms in $\mathcal{B} Q_{n, 2}$ has been considered by Calderon :

Theorem 3.1 ( $\mathrm{Ca})$. Let $F(x ; s, t)=a(x) s^{2}+2 b(x) s t+c(x) t^{2} \in \mathcal{B} Q_{n, 2}$ where $x=\left(x_{0}, \ldots, x_{n-1}\right)$ and $s, t$ are real variables.

If $F$ is positive semi-definite, then it is a sum of squares. Namely, there exists an integer $N$ and some linear forms $u_{i}(x), v_{i}(x)$ in $x$ such that

$$
F(x ; s, t)=\sum_{i=1}^{N}\left(u_{i}(x) s+v_{i}(x) t\right)^{2} .
$$

The original proof of Calderon is based on a convexity argument. We will give another proof without convexity. The advantages are that it yields an effective construction of the sum of squares and gives a better bound on the number of needed squares. Moreover, we will deal with very similar constructions in the following of the paper.

Our proof can be thought as a constructive version of [Ca], in the same way as $[\mathrm{Pf}]$ is a constructive version of original Hilbert's Theorem on ternary quartics.

Proof. Let us assume first that $F$ is positive definite, namely for all $x \neq 0$ and all $(s, t) \neq(0,0), F(x ; s, t)>0$.

Let

$$
F_{\epsilon}(x ; s, t)=F(x ; s, t)-\epsilon x_{0}^{2} s^{2}
$$

and introduce $I=\left\{\epsilon \mid F_{\epsilon} \in \mathcal{P} Q_{n, 2}\right\}$. Then, $I$ is a non empty interval $[0, m)$. Since $I$ is defined by some closed conditions, we have $I=[0, m]$. Necessarily $m \neq 0$ and $F_{m}$ is not positive definite.

Hence, we have reduced to the case where $F$ is not positive definite : there is $\left(s_{0}, t_{0}\right) \neq(0,0)$ and $X_{0} \neq 0$ such that $F\left(X_{0} ; s_{0}, t_{0}\right)=0$.

Up to a linear change of the coordinates we may assume that $\left(s_{0}, t_{0}\right)=(0,1)$ and $X_{0}=(1,0, \ldots, 0)$. Then, there are some identities between quadratic forms : 


$$
\left\{\begin{array}{l}
a(x)=\left(\lambda x_{0}+\alpha(y)\right)^{2}+\widetilde{a}(y) \\
b(x)=\eta x_{0}^{2}+\left(\lambda x_{0}+\alpha(y)\right) \beta(y)+\widetilde{b}(y) \\
c(x)=\mu\left(x_{0}+\gamma(y)\right)^{2}+\widetilde{c}(y)
\end{array}\right.
$$

Where $\lambda, \mu, \eta$ are reals, $\alpha(y), \beta(y), \gamma(y)$ are linear forms and $\widetilde{a}(y), \widetilde{b}(y), \widetilde{c}(y)$ are quadratic forms in the variables $y=\left(x_{1}, \ldots, x_{n-1}\right)$.

By assumption we have $\mu=0$. By positivity of $F$, we get $\eta=0$. Thus

$$
F(x ; s, t)=\left[\left(\lambda x_{0}+\alpha(y)\right) s+\beta(y) t\right]^{2}+\widetilde{F}(y ; s, t)
$$

where

$$
\widetilde{F}(y ; s, t)=\widetilde{a}(y) s^{2}+2 \widetilde{b}(y) s t+\left(\widetilde{c}(y)-\beta(y)^{2}\right) t^{2} .
$$

Since $F$ is positive semi-definite, we deduce that $\widetilde{F}$ is also positive semi-definite.

Then, by induction on the number of variables $n$, we conclude that $F$ is a sum of squares.

Moreover, at each step we get rid of one of the variables $x_{i}$ and we increase by two the needed number of squares to decompose our positive semi-definite biquadratic form. It gives the bound $N \leq 2 n$.

Let us mention also that Theorem 3.1 can also be seen as a particular case of [CLR1, Theorem 7.1], which provides another different proof.

3.2. On the number of squares. It is common in real algebra to study the minimal number of squares in a decomposition of a given sum of squares.

For positive semi-definite biquadratic forms in $\mathcal{B} Q_{n, 2}$, our proof of Theorem 3.1 gives the bound $N \leq 2 n$, whereas the original argument of Calderon who relies on a convexity argument leads to $N \leq 3 n(n+1) / 2$ squares.

By [CLR1, Theorem 7.1], we also recover the bound $2 n$, but in fact it is possible to do even better. The needed number of squares is showed in [CLR1] to be bounded by

$$
\left\lfloor\sqrt{3} n+\frac{\sqrt{3}-1}{2}\right\rfloor .
$$

In general, if $F \in \mathcal{B} Q_{n, m}$ is assumed to be a sum of squares, then according to the work in [CLR2] about the so-called pythagoras numbers, one can say that $F$ is a sum of at most

$$
\frac{(\sqrt{1+2 m n(m+1)(n+1)}-1)}{2}
$$

squares.

See also [LS] for other bounds.

\section{Counting the Roots of a Biquadratic Form}

In CLR1 is defined $B_{n, k}$ (resp. $\left.B_{n, k}^{\prime}\right)$ by $\sup |\mathcal{Z}(p)|$ where $p$ ranges over all positive semi-definite forms in $\mathcal{H}_{n, k}$ (resp. sum of squares of forms) with $|\mathcal{Z}(p)|<$ 
$\infty$. The determination of these numbers is a rather awesome task. Some partial results are given in CLR1. For instance :

$$
\left\{\begin{array}{l}
B_{3,4}=4, \\
B_{3,6}=10, \\
\text { For } k>6, \frac{k^{2}}{4} \leq B_{3, k} \leq \frac{(k-1)(k-2)}{2} \quad \text { and } \quad B_{3, k}^{\prime}=\frac{k^{2}}{4} .
\end{array}\right.
$$

Note that in general, we do not even know if the numbers $B_{n, k}$ are finite.

We naturally introduce the counterpart for biquadratic forms. Namely, let $\mathrm{BB}_{n, m}$ (resp. $\quad \mathrm{BB}_{n, m}^{\prime}$ ) be equal to $\sup |\mathcal{Z}(F)|$ where $F$ ranges over all forms in $\mathcal{P} Q_{n, m}$ (resp. sum of squares of forms) with $|\mathcal{Z}(F)|<\infty$.

Proposition 4.1. We have:

a) $\mathrm{BB}_{n, m} \geq \mathrm{BB}_{n, m}^{\prime}$,

b) $\mathrm{BB}_{n, 2}=\mathrm{BB}_{n, 2}^{\prime}$,

c) $\mathrm{BB}_{n, m}=\mathrm{BB}_{m, n}$,

d) $\mathrm{BB}_{n_{1}+n_{2}, m_{1}+m_{2}} \geq \mathrm{BB}_{n_{1}, m_{1}}+\mathrm{BB}_{n_{2}, m_{2}}$,

e) $\mathrm{BB}_{n, m} \leq B_{n, 2 m} \cdot B_{m, 2 n}$.

Moreover, $(c)$ and $(d)$ remain true if $\mathrm{BB}$ is replaced everywhere by $\mathrm{BB}^{\prime}$.

Proof. Properties (a) and (c) are obvious, and (b) follows from Theorem 3.1.

To show property $(\mathrm{d})$, we take two biquadratic forms

$$
F_{1}(x ; s) \in \mathcal{P} Q_{n_{1}, m_{1}} \text {, with } x=\left(x_{0}, \ldots, x_{n_{1}-1}\right), s=\left(s_{0}, \ldots, s_{m_{1}-1}\right)
$$

and

$$
F_{2}(y ; t) \in \mathcal{P} Q_{n_{2}, m_{2}} \text { with } y=\left(y_{0}, \ldots, y_{n_{2}-1}\right), t=\left(t_{0}, \ldots, t_{m_{2}-1}\right)
$$

Then, consider the biquadratic form

$$
F(x, y ; s, t)=F_{1}(x ; s)+F_{2}(y ; t)
$$

which is in $\mathcal{P} Q_{n_{1}+n_{2}, m_{1}+m_{2}}$.

Let us show now property (e). Let $F(x ; s) \in \mathcal{P} Q_{n, m}$ and let us introduce $p(x)=$ $\operatorname{det}\left(M_{x}(F)\right) \in \mathcal{H}_{n, 2 m}$ and $q(s)=\operatorname{det}\left(M_{s}(F)\right) \in \mathcal{H}_{m, 2 n}$. They are both positive semi-definite. We recall from Proposition 2.2 that if $P \in \mathcal{Z}(F)$, then $\Pi_{1}(P)$ is a zero of $p(x)$ and $\Pi_{2}(P)$ is a zero of $q(s)$. Then, $\left|\Pi_{1}(\mathcal{Z}(F))\right| \leq B_{n, 2 m}$ and $\left|\Pi_{2}(\mathcal{Z}(F))\right| \leq$ $B_{m, 2 n}$. This concludes the proof.

In the sequel, we compute the values of $\mathrm{BB}_{n, m}^{\prime}$, using the notion of degree of an algebraic variety embedded in a projective space.

Let us consider the Segre embedding

$$
\begin{array}{ccc}
\Phi: \mathbb{P}^{n-1} \times \mathbb{P}^{m-1} & \rightarrow & \mathbb{P}^{n m-1} \\
\left(\left[x_{0}, \ldots, x_{n-1}\right],\left[s_{0}, \ldots, s_{m-1}\right]\right) & \rightarrow & {\left[x_{0} s_{0}, x_{0} s_{1}, \ldots, x_{n-1} s_{m-1}\right]}
\end{array}
$$

Let us recall (see [Ha] for instance) that if the algebraic variety $V \subset \mathbb{P}^{n}$ has dimension $r$, then its degree $d$ is equal to the number of intersection points with a generic linear variety in $\mathbb{P}^{n}$ of dimension $n-r$. Moreover, the number of intersection points of $V$ with any linear variety in $\mathbb{P}^{n}$ of dimension $n-r$, is then at most $d$. Further, the number of intersection points of $V$ with any linear variety in $\mathbb{P}^{n}$ of dimension $>n-r$, is at most $d$ or infinite.

We have :

Theorem 4.2. $\mathrm{BB}_{n, m}^{\prime}=\left(\begin{array}{c}m+n-2 \\ m-1\end{array}\right)$. 
Proof. Let $F \in \mathcal{P} Q_{n, m}$ such that $F=\sum_{i=1}^{r} F_{i}^{2}$. Let us write

$$
F_{i}=\sum_{0 \leq j \leq n-1,0 \leq k \leq m-1} a_{j, k ; i} x_{j} s_{k}
$$

and let us consider the hyperplane $H_{i}$ in $\mathbb{P}^{m n-1}$ of equation

$$
\sum_{0 \leq j \leq n-1,0 \leq k \leq m-1} a_{j, k ; i} t_{j, k}=0 .
$$

Then, $F(P)=0$ if and only if $F_{1}(P)=\ldots=F_{r}(P)=0$. In other words, $\Phi(P)$ belongs to the linear space $H$ defined by $H=\cap_{i} H_{i}$. Namely

$$
|\mathcal{Z}(F)|=\left|\left\{P \in \mathbb{P}^{n-1} \times \mathbb{P}^{m-1} \mid \forall i F_{i}(P)=0\right\}\right|=|\operatorname{Im} \Phi \cap H| .
$$

According to [Ha], the dimension of $\operatorname{Im} \Phi$ is $m+n-2$ and the degree of the embedding $\Phi$ is $\left(\begin{array}{c}n+m-2 \\ n-1\end{array}\right)$ (this is true over any field of characteristic zero). The degree of $\Phi$ being precisely the maximal number of intersection points of $\operatorname{Im} \Phi$ with a linear space such that the intersection is finite, we are done.

Example 4.3. In the case $n=m=3$, we have $6=\mathrm{BB}_{3,3}^{\prime}$. Here are two examples $F_{1}$ and $F_{2}$ of biquadratic forms in $\mathcal{P} Q_{3,3}$ with the maximal umber of real zeros:

a)

$$
F_{1}(x, y, z ; s, t, u)=(x s)^{2}+(y t)^{2}+(z u)^{2}+((x+y+z)(s+t+u))^{2} .
$$

b)

$$
M_{(x, y, z)}\left(F_{2}\right)=\left(\begin{array}{ccc}
x^{2}+y^{2}+z^{2} & x y+z^{2} & x z+y^{2} \\
x y+z^{2} & x^{2}+y^{2}+z^{2} & y z+x^{2} \\
x z+y^{2} & y z+x^{2} & x^{2}+y^{2}+z^{2}
\end{array}\right) .
$$

In both cases, the set of real zeros is

$$
\left\{\begin{array}{lll}
(1,0,0 ; 0,-1,1), & (0,1,0 ; 1,0,-1), & (0,0,1 ;-1,1,0), \\
(1,-1,0 ; 1,1,-1), & (0,1,-1 ;-1,1,1), & (-1,0,1 ; 1,-1,1)
\end{array}\right\} .
$$

As a direct consequence of the definition of the numbers $\mathrm{BB}, \mathrm{BB}^{\prime}$ and Theorem 4.2. we have

Proposition 4.4. Let $F \in \mathcal{P} Q_{n, m}$ be such that $\mathrm{BB}_{n, m}^{\prime}<|\mathcal{Z}(F)| \leq \mathrm{BB}_{n, m}$. Then, $F$ is not a sum of squares. For instance, if $F \in \mathcal{P} Q_{3,3}$ is such that $6<|\mathcal{Z}(F)|<\infty$, then $F$ is not a sum of squares.

\section{Zeros OF BIQUADRATIC FORMS IN $\mathcal{B} Q_{3,3}$}

The main purpose of this section is to show that if a biquadratic form in $\mathcal{P} Q_{3,3}$ has more zeros than $\mathrm{BB}_{3,3}$, then it is a sum of squares (Theorem 5.4). It is a kind of counterpart for biquadratic forms of the property [CLR1, Theorem 3.5] for positive semi-definite forms in $\mathcal{H}_{3, k}$.

Although, some differences remain between these two contexts. For instance, if a positive semi-definite form in $\mathcal{H}_{3, k}$ has an infinite number of zeros, then it is divisible by the square of some indefinite form. Whereas, for biquadratic forms, it is no more true. Indeed, let us consider

$$
F(x, y, z ; s, t, u)=(x s)^{2}+(y t)^{2} \in \mathcal{P} Q_{3,3} .
$$


It has an infinite number of zeros (for instance $(0,0,1 ; s, t, u)$, for any $\left.(s, t, u) \in \mathbb{P}^{2}\right)$, but cannot be divided by a bilinear form.

We are mainly interested in the case of the dimension 2 , since it is the easiest case where positive semi-definite biquadratic forms do not coincide with sums of squares. Besides, in higher dimensions, such a property is hopeless as it is shown by :

Example 5.1. Let $F(x, y, z ; s, t, u)$ be a positive semi-definite non sum of squares biquadratic form in $\mathcal{B} Q_{3,3}$. Assume that $(1,0,0 ; 1,0,0)$ is a zero of $F$ in $\mathbb{P}^{2} \times \mathbb{P}^{2}$ and let $L(x, y, z)$ be a non zero linear form such that $L(1,0,0)=0$. Then, the following biquadratic form

$$
G(x, y, z ; s, t, u, v)=F(x, y, z ; s, t, u)+L(x, y, z)^{2} v^{2} \in \mathcal{P} Q_{3,4}
$$

is not a sum of squares. Moreover, $G$ has an infinite number of zeros in $\mathbb{P}^{2} \times \mathbb{P}^{3}$, for instance $(1,0,0 ; 1,0,0, v)$ for any $v \in \mathbb{R}$.

That is why, in the following, we cannot reproduce the whole method of CLR1, although some steps will look very similar.

Let us begin with

Lemma 5.2. Let $F \in \mathcal{P} Q_{3,3}$ be a positive semi-definite biquadratic form which admits two different zeros $A$ and $B$ in $\mathbb{P}^{2} \times \mathbb{P}^{2}$ such that $\Pi_{1}(A)=\Pi_{1}(B)$. Then, $F$ is a sum of squares and admits an infinite number of zeros.

Proof. After a linear change of coordinates, we may assume that $A=(1,0,0 ; 1,0,0)$ and $B=(1,0,0 ; 0,1,0)$. Denoting by $(x, y, z ; s, t, u)$ the new coordinates, the biquadratic form $F$ can be written as

$F(x, y, z ; s, t, u)=a_{3,3}(x, y, z) u^{2}+2 a_{1,3}(x, y, z) s u+2 a_{2,3}(x, y, z) t u+G(y, z ; s, t, u)$

where $G$ is a biquadratic form and $a_{1,3}, a_{2,3}$ and $a_{3,3}$ are quadratic forms. If $a_{3,3}$ does not depend on the variable $x$, then it is the same for $a_{1,3}$ and $a_{2,3}$, namely $F$ does not depend on $x$. In that case, $(\alpha, 0,0 ; 1,0,0)$ would be a zero of $F$ for any $\alpha \in \mathbb{R}$, and $F$ would be a sum of squares by Theorem 3.1.

In the following, we assume that $a_{3,3}$ does depend on $x$. We may rewrite $F$ as

$$
F(x, y, z ; s, t, u)=a_{3,3}(x, y, z) u^{2}+2 x \alpha_{1}(y, z) s u+2 x \alpha_{2}(y, z) t u+H(y, z ; s, t, u)
$$

where $H$ is a biquadratic form and $\alpha_{1}$ and $\alpha_{2}$ are linear forms. Up to a linear change of the coordinate $x$, we may assume that $a_{3,3}(x, y, z)=x^{2}+b_{3}(y, z)$ where $b_{3}$ is a quadratic form.

We have then

$$
F(x, y, z ; s, t, u)=\left(x u+\alpha_{1} s+\alpha_{2} t\right)^{2}+\widetilde{F}(y, z ; s, t, u)
$$

where $\widetilde{F}$ is a biquadratic form.

Since $F$ is positive semi-definite, so it is the case for $\widetilde{F}$. Then, $\widetilde{F}$ is a sum of squares by Theorem 3.1. Moreover, $(1,0,0 ; \alpha, \beta, 0)$ is a zero of $F$ for any $(\alpha, \beta) \in$ $\mathbb{R}^{2}$.

As a consequence, we have 
Proposition 5.3. Let $F \in \mathcal{P} Q_{3,3}$ be a positive semi-definite biquadratic form with at least 11 zeros in $\mathbb{P}^{2} \times \mathbb{P}^{2}$. Then, $F$ admits an infinite number of zeros. In other words, $\mathrm{BB}_{3,3} \leq 10$.

Proof. Let us consider the associated matrix $M_{(s, t, u)}(F)$ whose entries are quadratic forms in the variables $(s, t, u)$. According to Lemma 5.2, we may assume that the number of zeros $\mathcal{Z}(F)$ of $F$ is equal to $\left|\Pi_{2}(\mathcal{Z}(F))\right|$. Then, the number of zeros of $F$ is the number of zeros in $\mathbb{P}^{2}$ of $\operatorname{det}\left(M_{(s, t, u)}(F)\right)$ by Proposition 2.2 .

According to [CLR1, Theorem 3.7], we deduce that $|\mathcal{Z}(F)|$ is either infinite or it is bounded by $B_{3,6}=10$.

Our purpose now is to show in addition that $F$ necessarily is a sum of squares.

Theorem 5.4. Let $F \in \mathcal{P} Q_{3,3}$ be a positive semi-definite biquadratic form with an infinite number of zeros in $\mathbb{P}^{2} \times \mathbb{P}^{2}$. Then, $F$ is a sum of squares.

Proof. Up to a linear change of coordinates, we may assume that $(1,0,0 ; 1,0,0)$ is an accumulation point in $\mathcal{Z}(F) \subset \mathbb{P}^{2} \times \mathbb{P}^{2}$.

Then,

$$
F(x, y, z ; s, t, u)=q_{2,0}(y, z) s^{2}+2 q_{2,1}(y, z ; t, u) s+q_{2,2}(x, y, z ; t, u)
$$

where the $q_{2, k}$ 's are quadratic forms in $(x, y, z)$ and forms of degree $k$ in $(s, t, u)$.

Note that necessarily $q_{2,0}$ and $q_{2,2}$ are positive semi-definite. Let us show a Lemma which is very close in spirit as [CLR1, Lemma 5.2] :

Lemma 5.5. With the above notations, we have $\operatorname{rk}\left(q_{2,0}\right)<2$.

Proof. Assume that $\operatorname{rk}\left(q_{2,0}\right)=2$. Up to a linear change of coordinates, we may assume that $q_{2,0}=y^{2}+z^{2}$. Consider a sequence $\left(1, y_{n}, z_{n} ; 1, t_{n}, u_{n}\right)$ of zeros of $F$ in $\mathbb{P}^{2} \times \mathbb{P}^{2}$ which converges to $(1,0,0 ; 1,0,0)$. We may assume that $\left(y_{n}, z_{n}\right) \neq(0,0)$ for all $n$ otherwise we may directly use Lemma 5.2 .

Let us choose an integer $n_{0}$ such that

$$
\frac{2\left|q_{2,1}\left(y_{n_{0}}, z_{n_{0}} ; t_{n_{0}}, u_{n_{0}}\right)\right|}{q_{2,0}\left(y_{n_{0}}, z_{n_{0}}\right)}<\frac{1}{2} .
$$

This is possible since, given $(a, b, c) \in \mathbb{R}^{2}$, the map

is bounded.

$$
\begin{array}{rlc}
\mathbb{P}^{1} & \longrightarrow & \mathbb{R} \\
(x, y) & \mapsto & \frac{a x^{2}+b x y+c y^{2}}{x^{2}+y^{2}}
\end{array}
$$

We have then

$$
F\left(1, y_{n_{0}}, z_{n_{0}} ; 1, t_{n_{0}}, u_{n_{0}}\right)=0 \geq q_{2,0}\left(y_{n_{0}}, z_{n_{0}}\right)-2\left|q_{2,1}\left(y_{n_{0}}, z_{n_{0}} ; t_{n_{0}} ; u_{n_{0}}\right)\right|>0
$$

a contradiction.

If $\operatorname{rk}\left(q_{2,0}\right)=0$, then we are done by Theorem 3.1. Hence, we are reduced to the case $\operatorname{rk}\left(q_{2,0}\right)=1$, i.e. after a linear change of coordinates, $q_{2,0}=y^{2}$.

We have

$$
F=y^{2} s^{2}+2 q_{2,1}(y, z ; t, u) s+q_{2,2}(x, y, z ; t, u),
$$

where $y^{2} q_{2,2}-q_{2,1}^{2}$, the discriminant of $F$ with respect to the variable $s$, is positive semi-definite. Hence, there is no coefficient $z^{2}$ in $q_{2,1}$, namely

$$
q_{2,1}=\alpha(t, u) y z+\beta(t, u) y^{2}
$$


where $\alpha, \beta$ are linear forms. We have

$$
F=(y s+\alpha(t, u) z+\beta(t, u) y)^{2}+G(x, y, z ; t, u)
$$

where $G$ is a biquadratic form.

Since, for any choice of $(y, x, z, t, u) \in \mathbb{R}^{*} \times \mathbb{R}^{4}$ there is a $s$ such that

$$
F(x, y, z ; s, t, u)=G(x, y, z ; t, u)
$$

we conclude that $G$ is also positive semi-definite. It suffices to uses Theorem 3.1 to conclude that $G$ is a sum of squares.

Remark 5.6. A little inspection of the proofs of 5.4 and 5.2 shows that if $F \in \mathcal{P} Q_{3,3}$ has an infinite number of zeros, then it is a sum of at most 5 squares. Indeed, when $F$ does not depend on $x$, we may consider that $F \in \mathcal{P} Q_{3,2}$ and we get the bound by (1).

We assume now that $F$ does depend on $x$, and we use equality (3). If $G$ had only a finite number of zeros, it would mean that $F$ had zeros of the form $\left(1, y_{n}, z_{n} ; 1,0,0\right)$, as a consequence $F$ would not depend on $s$ and we are done by the previous case.

Hence, we may assume that $G$ has an infinite number of zeros and we repeat the same argument to the biquadratic form $G$.

We have to successively use the bounds given by (11): it gives at most 4 squares for $\mathcal{B} Q_{3,2}$, at most 3 squares for $\mathcal{B} Q_{2,2}$ and at most 2 squares for $\mathcal{B} Q_{2,1}$.

In summary, the maximal number of needed squares in all sums of squares lying in $\mathcal{B} Q_{3,3}$ is at most 8 according to (2). But when we restrict to those sum of squares with an infinite number of zeros, the bound drops to 5 .

At least, we cannot expect less than 5 squares as shown by the following example

$$
F=x^{2} s^{2}+y^{2} t^{2}+\left(x^{2}+y^{2}+z^{2}\right) u^{2} \text {. }
$$

\section{EXAmples of POSITIVE SEMI-DEFInite BIQUADRATIC FORMS Which ARE NOT SUM OF SQUARES}

Positive semi-definite biquadratic forms which are not sums of squares have some important applications in several areas such as control theory and theoretical physics, even if in that last case the complex counterpart of theses notions are most commonly studied, namely positive semi-definite bihermitian forms which are not sums of squares (see for instance $[\mathrm{SZ}]$ ).

In this section, we mention some known examples given in the literature, for instance in [C1] and [Te]. We revisit them by making use of the notion of real zeros of biquadratic forms we have developed in the previous sections.

To end, we will also produce some new families of examples.

6.1. Arrow matrices. If $F(x ; s) \in \mathcal{B} Q_{3,3}$ has some associated matrix $M_{x}(F)$ with more than two zero entries, then it obviously is a sum of squares. Hence, the simplest candidate for an example of a positive semi-definite biquadratic form not being a sum of squares is an arrow matrix. In this section, we show that such examples exist.

Proposition 6.1. The biquadratic form $F_{\alpha}$ whose associated matrix is

$$
M_{\alpha}=\left(\begin{array}{ccc}
x^{2}+z^{2} & 0 & -x z \\
0 & x^{2}+y^{2} & -y z \\
-x z & -y z & z^{2}+\alpha y^{2}
\end{array}\right)
$$


is positive semi-definite but not a sum of squares for all $\alpha \in\left[\alpha_{0},+\infty\left[\right.\right.$, where $\alpha_{0}$ is a smallest positive root of the polynomial $9 x^{4}-36 x^{3}+62 x^{2}-20 x+1\left(\alpha_{0} \simeq 0.006\right)$.

Proof. The matrix $M_{\alpha}$ is positive semi-definite if and only if all its symmetric minors are positive semi-definite. The only non trivial condition to check is $\operatorname{det}\left(M_{\alpha}\right) \geq 0$. Let us compute

$$
\operatorname{det}\left(M_{\alpha}\right)=\alpha y^{2} x^{4}+\left(\alpha y^{4}+(\alpha-1) y^{2} z^{2}+z^{4}\right) x^{2}+\alpha y^{4} z^{2} .
$$

An elementary computation shows that $\operatorname{det}\left(M_{\alpha}\right) \geq 0$ if and only if $\alpha \in\left[\alpha_{0},+\infty[\right.$.

Now, let us see why $F_{\alpha}$ is not a sum of squares. We have :

$$
F_{\alpha}=\left(x^{2}+z^{2}\right) s^{2}+\left(x^{2}+y^{2}\right) t^{2}+\left(z^{2}+\alpha y^{2}\right) u^{2}-2 x z s u-2 y z t u
$$

If we look at $F_{\alpha}$ as a form of degree 4 in the 6 variables $(x, y, z, s, t, u)$, then we may consider its associated Gram matrices (see CLR2 for background on Gram Matrices and sums of squares) with respect to the basis $(x s, x t, y t, y u, z s, z u)$. We find that there is a unique Gram matrix :

$$
G_{\alpha}=\left(\begin{array}{cccccc}
1 & 0 & 0 & 0 & 0 & -1 \\
0 & 1 & 0 & 0 & 0 & 0 \\
0 & 0 & 1 & 0 & 0 & -1 \\
0 & 0 & 0 & \alpha & 0 & 0 \\
0 & 0 & 0 & 0 & 1 & 0 \\
-1 & 0 & -1 & 0 & 0 & 1
\end{array}\right) .
$$

Its signature is $(5,1)$ whenever $\alpha>0$. Hence $G_{\alpha}$ is never a positive semi-definite matrix, and $F_{\alpha}$ is never a sum of squares when $\alpha>0$.

In this example, each associated matrices $M_{(x, y, z)}\left(F_{\alpha}\right)$ and $M_{(s, t, u)}\left(F_{\alpha}\right)$ of the biquadratic form $F_{\alpha}$ are both arrow matrices.

We end this section by giving another example of an arrow matrix associated to a positive semi-definite biquadratic form which is not a sum of squares. Namely :

$$
\left(\begin{array}{ccc}
x^{2}+z^{2} & 0 & -x z-x y \\
0 & x^{2}+y^{2} & -y z \\
-x z-x y & -y z & 2 y^{2}+2 z^{2}
\end{array}\right) .
$$

6.2. Terpstra's construction. A geometric construction of positive semi-definite biquadratic forms which are not sum of squares can be found in $[\mathrm{Te}]$. For the convenience of the reader, we will recall the construction, although we give a different proof from the original, making use of our previous results on the real zeros set of biquadratic forms.

Let us consider $L_{1}, L_{2}, L_{3}, L_{4}$, four lines in general position in the projective plane $\mathbb{P}^{2}$. Let us introduce the intersection points

$$
\begin{aligned}
& A_{1}=L_{1} \cap L_{2}, \\
& A_{2}=L_{2} \cap L_{3}, \\
& A_{3}=L_{2} \cap L_{4}, \\
& A_{4}=L_{1} \cap L_{4}, \\
& A_{5}=L_{1} \cap L_{3}, \\
& A_{6}=L_{3} \cap L_{4} .
\end{aligned}
$$


Up to multiplying the equations by -1 , we may assume that $L_{1}\left(A_{6}\right) \cdot L_{2}\left(A_{6}\right)>0$ and $L_{3}\left(A_{1}\right) \cdot L_{4}\left(A_{1}\right)>0$. Let us define the following points in $\mathbb{P}^{2} \times \mathbb{P}^{2}$ :

$$
\begin{aligned}
& P_{1}=\left(A_{1}, A_{6}\right) \\
& P_{2}=\left(A_{2}, A_{4}\right) \\
& P_{3}=\left(A_{3}, A_{5}\right) \\
& P_{4}=\left(A_{4}, A_{2}\right) \\
& P_{5}=\left(A_{5}, A_{3}\right) \\
& P_{6}=\left(A_{6}, A_{1}\right) .
\end{aligned}
$$

Theorem 6.2. Let us consider the biquadratic form

$$
\begin{aligned}
F_{\lambda}(x ; s)= & \left(L_{1}(x) L_{1}(s)\right)^{2}+\left(L_{2}(x) L_{2}(s)\right)^{2}+\left(L_{3}(x) L_{3}(s)\right)^{2}+\left(L_{4}(x) L_{4}(s)\right)^{2} \\
& +\lambda L_{1}(x) L_{2}(x) L_{3}(s) L_{4}(s)
\end{aligned}
$$

where $\lambda \in \mathbb{R}$.

Then,

a) There is a $\delta>0$ such that for all $0<\lambda<\delta$, the biquadratic form $F_{\lambda}$ is positive semi-definite,

b) For any $\lambda>0$, the biquadratic form $F_{\lambda}$ is never a sum of squares.

Proof. One easily checks that $F_{\lambda}\left(P_{1}\right)=\ldots=F_{\lambda}\left(P_{5}\right)=0$ and $F_{\lambda}\left(P_{6}\right)>0$.

Let us show that $F_{\lambda} \geq 0$ in a neighbourhood of each $P_{i}$ for $i=1, \ldots, 5$ (it is obvious for $i=6$ since $\left.F_{\lambda}\left(P_{6}\right)>0\right)$. Let

$$
\begin{aligned}
F_{2, \delta}(x ; s)= & L_{1}^{2}\left(A_{2}\right) L_{1}^{2}(s)+L_{2}^{2}\left(A_{4}\right) L_{2}^{2}(x)+L_{3}^{2}\left(A_{2}\right) L_{3}^{2}(x)+L_{4}^{2}\left(A_{2}\right) L_{4}^{2}(s) \\
& +\delta L_{1}\left(A_{2}\right) L_{3}\left(A_{4}\right) L_{2}(x) L_{4}(s)
\end{aligned}
$$

Since $F_{\lambda}-F_{2, \delta}$ is infinitely smaller than $F_{2, \delta}$ in a small neighbourhood of $P_{2}$, it suffices to note that $F_{2, \delta}>0$ in a neighbourhood of $P_{2}$ for $\delta$ small enough.

We proceed likewise in a neighbourhood of $P_{3}, P_{4}$ and $P_{5}$.

Around $P_{1}$, we also proceed likewise but the expression we consider is a little bit different : we set

$$
F_{1}(x ; s)=L_{1}^{2}\left(A_{6}\right) L_{1}^{2}(x)+L_{2}^{2}\left(A_{6}\right) L_{2}^{2}(x)+L_{3}^{2}\left(A_{1}\right) L_{3}^{2}(s)+L_{4}^{2}\left(A_{1}\right) L_{4}^{2}(s)
$$

which is $>0$ in a small neighbourhood of $P_{1}$.

In summary, there is a $\delta>0$ and an open neighbourhood $V$ of $P_{1}, P_{2}, P_{3}, P_{4}, P_{5}$ such that $F_{\lambda}>0$ on $V \backslash\left\{P_{1}, P_{2}, P_{3}, P_{4}, P_{5}\right\}$ for any $0<\lambda<\delta$.

On $\Omega=\mathbb{P}^{2} \backslash V$, the quantity

$$
\frac{\left(L_{1}(x) L_{1}(s)\right)^{2}+\left(L_{2}(x) L_{2}(s)\right)^{2}+\left(L_{3}(x) L_{3}(s)\right)^{2}+\left(L_{4}(x) L_{4}(s)\right)^{2}}{\left|L_{1}(x) L_{2}(x) L_{3}(s) L_{4}(s)\right|}
$$

never vanishes, hence reaches a minimum which we may assume greater than $\delta$.

We have shown that $F_{\lambda}$ is positive semi-definite for any $0<\lambda<\delta$.

Let us now show that $F_{\lambda}$ is never a sum of squares.

By the contrary, let us assume that $F=\sum_{i=1}^{r} G_{i}^{2}$ where $G_{i}$ is a bilinear form in the variables $(x ; s)$.

Let us consider the linear map which sends a bilinear map $G$ to the 6-tuple $\left(G\left(P_{1}\right), \ldots, G\left(P_{6}\right)\right)$. The range of this map is contained in an hyperplane of $\mathbb{R}^{6}$. Namely, there are some real numbers $\alpha_{1}, \ldots \alpha_{6}$ such that for any bilinear form $G$, we have

$$
\alpha_{1} G\left(P_{1}\right)+\ldots+\alpha_{6} G\left(P_{6}\right)=0
$$


Let us assume that $\alpha_{6}=0$. We consider the bilinear form $G=L_{2}(x) L_{3}(s)$. It vanishes at $P_{1}, P_{2}, P_{3}, P_{4}$ but not at $P_{5}, P_{6}$, and hence we get $\alpha_{5} G\left(P_{5}\right)=0$. Thus, $\alpha_{5}=0$ and we shall proceed likewise to show that all $\alpha_{i}$ are zero, a contradiction. Then, necessarily $\alpha_{6} \neq 0$.

Now, it suffices to notice that we obviously have, for all $i$,

$$
G_{i}\left(P_{1}\right)=\ldots=G_{i}\left(P_{5}\right)=0 .
$$

Hence $G_{i}\left(P_{6}\right)=0$ for all $i$ which implies that $F\left(P_{6}\right)=0$, a contradiction.

Note that, according to the construction, $\mathcal{Z}\left(F_{\lambda}\right)=\left\{P_{1}, \ldots, P_{6}\right\}$ and that the relation $\alpha_{1} G\left(P_{1}\right)+\ldots+\alpha_{6} G\left(P_{6}\right)=0$ can be seen as Cayley-Bacharah relation (EGH).

\subsection{Around the Choi example.}

6.3.1. The Choi example. As defined in [C1], we consider the following biquadratic form :

$C_{1}(x, y, z ; s, t, u)=\left(x^{2}+2 z^{2}\right) s^{2}+\left(y^{2}+2 x^{2}\right) t^{2}+\left(z^{2}+2 y^{2}\right) u^{2}-2 x y s t-2 x z s u-2 y z t u$

Denote by $\mathcal{C}$ its zero set $\mathcal{Z}\left(C_{1}\right)$ in $\mathbb{P}^{2} \times \mathbb{P}^{2}$. We have

$$
\mathcal{C}=\left\{A_{1}, A_{2}, A_{3}, B_{0}, B_{1}, B_{2}, B_{3}\right\}
$$

where

$$
\begin{aligned}
& A_{1}=(1,0,0 ; 0,1,0), A_{2}=(0,1,0 ; 0,0,1), A_{3}=(0,0,1 ; 1,0,0), B_{0}=(1,1,1 ; 1,1,1) \\
& B_{1}=(-1,1,1 ;-1,1,1), B_{2}=(1,-1,1 ; 1,-1,1), B_{3}=(1,1,-1 ; 1,1,-1) .
\end{aligned}
$$

Proposition 6.3. The biquadratic form $C_{1}$ is positive semi-definite but not a sum of squares.

We will not discuss much the fact that $C_{1}$ is positive semi-definite. For instance, it comes from the property that all the symmetric minors of $M_{(x, y, z)}\left(C_{1}\right)$ are sums of squares.

The original proof to show that $C_{1}$ is not a sum of squares is rather elementary : we assume that $C_{1}$ is a sum of squares and we carefully look at the monomials in $C_{1}$ to get a contradiction.

Now, referring to our background on real zeros of biquadratic forms, the quickest way to reprove it, is to use Proposition 4.4. But let us show again another proof of that fact!

Our motivation for this new proof is that it might lead to another direction to study positive semi-definite biquadratic forms which are not sum of squares. This proof is close in spirit than the one Robinson used (a strategy suggested by Hilbert's work) to show that its polynomial

(5) $R(x, y, z)=x^{6}+y^{6}+z^{6}-x^{4} y^{2}-x^{4} z^{2}-y^{4} x^{2}-y^{4} z^{2}-z^{4} x^{2}-z^{4} y^{2}+3 x^{2} y^{2} z^{2}$

is positive semi-definite but not a sum of squares. In modern literature, it would be referred as a Cayley-Bacharah type result (see EGH]). Namely, it exploits the redundancy that exists among the real zeros of cubic forms, redundancy which is looser among the real zeros of sextic forms.

Our proof could be seen as a counterpart for biquadratic forms, namely a CayleyBacharah type result for biquadratic forms : 
Proof. Assume that $C_{1}=\sum_{i=1}^{r} f_{i}^{2}$. The bilinear form $f_{i}$ can be written

$$
f_{i}=a_{1} x s+a_{2} x t+a_{3} x u+b_{1} y s+b_{2} y t+b_{3} y u+c_{1} z s+c_{2} z t+c_{3} z u .
$$

Since each $f_{i}$ has to vanish at $A_{1}, A_{2}, A_{3}$, we deduce that $a_{2}=b_{3}=c_{1}=0$.

Moreover, if we write the system saying that $f_{i}$ vanishes also at $B_{0}, B_{1}, B_{2}, B_{3}$, we conclude that

$$
f_{i}=a_{1} x s+b_{2} y t+c_{3} z u \quad \text { with } \quad a_{1}+a_{2}+a_{3}=0
$$

As a consequence, we get that each $f_{i}$ vanishes also at $A_{\alpha}=(0,1, \alpha ; 1,0,0)$ for any $\alpha \in \mathbb{R}$. And hence $C_{1}$ should also vanishes at all $A_{\alpha}$, a contradiction.

In fact, we have shown that :

Proposition 6.4. ?? If $G$ is a biquadratic form which vanishes at $\mathcal{C}$ and not at $A_{\alpha}=(0,1, \alpha ; 1,0,0)$ for some $\alpha \in \mathbb{R}$, then $G$ is not a sum of squares.

Example 6.5. As an application, if $G$ is a positive semi-definite biquadratic form which vanishes at $\mathcal{C}$, then $C_{1}+G$ is positive semi-definite but not a sum of squares.

For instance :

$$
C_{1}+(2 x s-y t+z u)^{2}
$$

or

$$
C_{1}+(x s-y t)^{2}+(x s-z u)^{2}+(y t-z u)^{2} .
$$

6.3.2. Some homotopy around the Choi example. First note, as observed in [Q]], that the biquadratic form associated to the matrix

$$
M_{0}=\left(\begin{array}{ccc}
x^{2}+z^{2} & -x y & -x z \\
-x y & x^{2}+y^{2} & -y z \\
-x z & -y z & y^{2}+z^{2}
\end{array}\right)
$$

is positive semi-definite but not a sum of squares for the same reasons than $C_{1}$ is.

Here is some homotopy around $M_{0}$ :

Proposition 6.6. The matrix

$$
\begin{aligned}
M_{a}= & M_{0}+a\left(\begin{array}{ccc}
x^{2}-z^{2} & 0 & 0 \\
0 & y^{2}-x^{2} & 0 \\
0 & 0 & z^{2}-y^{2}
\end{array}\right) \\
= & \left(\begin{array}{ccc}
x^{2}+z^{2}+a\left(x^{2}-z^{2}\right) & -x y & -x z \\
-x y & x^{2}+y^{2}+a\left(y^{2}-x^{2}\right) & -y z \\
-x z & -y z & y^{2}+z^{2}+a\left(z^{2}-y^{2}\right)
\end{array}\right)
\end{aligned}
$$

is positive semi-definite for all $a \in[0,1]$. The associated biquadratic form $F_{a}$ has zero set $\mathcal{C}$, and it is positive semi-definite but not a sum of squares until $a=1$.

The easiest way to prove $M_{a}$ is not a sum of squares it is to use Proposition ?? : indeed $M_{a}(0,1,1 ; 1,0,0)=1-a \not 0$ if $a \in[0,1[$. But, we will give another proof, where appear some new plane ternary sextics which are positive semi-definite and not sums of squares (they are realized by a kind of homotopy between the two symmetric versions of the Motzkin polynomial).

Proof. The $1 \times 1$ minors of $M_{a}$ are positive semi-definite forms if and only if $-1 \leq$ $a \leq 1$. Adding the conditions given by the $2 \times 2$ minors, we get that $a \in[0,1]$. To end, we compute

$$
p_{a}=\operatorname{det}\left(M_{a}\right)=\alpha\left(y^{2} x^{4}+y^{4} z^{2}+x^{2} z^{4}\right)+\beta\left(x^{4} z^{2}+y^{4} x^{2}+z^{4} y^{2}\right)-3 \gamma x^{2} y^{2} z^{2}
$$


where

$$
\left\{\begin{array}{l}
\alpha=1-a-a^{2}+a^{3}, \\
\beta=2 a-a^{2}-a^{3}, \\
\gamma=1+a-2 a^{2} .
\end{array}\right.
$$

Our aim is to show that $p_{a}(x, y, z)$ is a positive semi-definite sextic but not a sum of squares. We will follow the same proof that is commonly used to show that the Motzkin polynomial is positive semi-definite but not a sum of squares.

First note that the arithmetico-geometric inequality gives $p_{a}(x, y, z) \geq 0$. Hence, $M_{a}$ is positive semi-definite if and only if $a \in[0,1]$.

Let us show now that the form $p_{a}(x, y, z)$ is not a sum of squares until $a=1$ (and then $\left.p_{a}=0\right)$. By the contrary assume that

$p_{a}=\sum_{i}\left(a_{i} x^{3}+b_{i} x^{2} y+c_{i} x^{2} z+d_{i} x y^{2}+e_{i} x y z+f_{i} x z^{2}+g_{i} y^{2}+h_{i} y^{2} z+k_{i} y z^{2}+l_{i} z^{3}\right)^{2}$.

We obviously have for all $i$,

$$
\left\{\begin{array}{l}
a_{i}=g_{i}=l_{i}=0 \\
\sum_{i} b_{i} c_{i}=\sum_{i} b_{i} d_{i}=\sum_{i} d_{i} h_{i}=0
\end{array}\right.
$$

Moreover, looking at the coefficient of $x^{2} y^{2} z^{2}$, we get

$$
\sum_{i} e_{i}^{2}+2 b_{i} k_{i}+2 c_{i} h_{i}+2 d_{i} f_{i}=-3 \gamma
$$

We have also

$$
\alpha=\sum b_{i}^{2}=\sum f_{i}^{2}=\sum h_{i}^{2}
$$

and

$$
\beta=\sum c_{i}^{2}=\sum d_{i}^{2}=\sum k_{i}^{2}
$$

Since $a \in[0,1]$, we have $\gamma \geq 0$ and by the Cauchy-Schwartz inequality, we deduce from (6) that

$$
3 \gamma+\sum_{i} e_{i}^{2} \leq 6 \alpha \beta
$$

Hence we get $\gamma-2 \alpha \beta \leq 0$ which is satisfied only if $a=1$.

The fact that $F_{a}$ is positive semi-definite and not a sum of squares for $a \neq 1$ is now a direct consequence of the Cauchy-Binet formula. Given matrices $A \in \mathbb{R}^{m \times s}$ and $B \in \mathbb{R}^{s \times m}$, the Cauchy-Binet formula states that

$$
\operatorname{det}(A B)=\sum_{S} \operatorname{det}\left(A_{S}\right) \operatorname{det}\left(B_{S}\right)
$$

where $S$ ranges over all the subsets of $\{1, \ldots s\}$ with $m$ elements, and $A_{S}$ (respectively $B_{S}$ ) denotes the matrix in $\mathbb{R}^{m \times m}$ whose columns are the columns of $A$ (respectively whose rows are the rows of $B$ ) with indexes from $S$.

If $F_{a}$ were a sum of squares, then we would have $M_{a}=A^{T} A$ for some matrix $A$ whose entries are linear forms in the variables $x, y, z$. Then,

$$
\begin{aligned}
\operatorname{det}\left(M_{a}\right) & =\sum_{S} \operatorname{det}\left(A^{T}\right)_{S} \operatorname{det}\left(A_{S}\right) \\
& =\sum_{S} \operatorname{det}\left(A_{S}^{T}\right) \operatorname{det}\left(A_{S}\right) . \\
& =\sum_{S} \operatorname{det}\left(A_{S}\right)^{2} .
\end{aligned}
$$

Which is a contradiction since $p_{a}$ is not a sum of squares. 


\section{Configuration of Zeros sets}

In order to study the possible geometry of the zeros sets of biquadratic forms in $\mathcal{P} Q_{n, m}$, we introduce the notion of maximal configurations which are finite subsets $\Omega \subset \mathbb{P}^{n-1} \times \mathbb{P}^{m-1}$ such that if $F \in \mathcal{P} Q_{n, m}$ vanishes at $\Omega$ and also at another point in $\mathbb{P}^{n-1} \times \mathbb{P}^{m-1}$, then $\mathcal{Z}(F)$ is infinite.

To begin with, one may look at what happens already for positive semi-definite forms.

7.1. Maximal configurations of Sextics. We say that $\Omega \subset \mathbb{P}^{n-1}$ is a maximal configurations if it is a finite subset $\Omega$ such that if $f \in \mathcal{H}_{n, k}$ is a positive semidefinite form which vanishes at $\Omega$ and also at another point in $\mathbb{P}^{n-1}$, then $\mathcal{Z}(f)$ is infinite.

From the definition of the number $B_{n, k}$, it follows that for any positive semidefinite $f \in \mathcal{H}_{n, k}$ such that $|\mathcal{Z}(f)|=B_{n, k}$, the set $\mathcal{Z}(f)$ is a maximal configuration.

For instance, the Robinson polynomial $R$ as defined in (5) gives an example of a 10-points maximal configuration since $|\mathcal{Z}(R)|=10$.

It is given as an open question in CLR1 whether it is possible to give examples of other (of all up to a linear change of coordinates ?) maximal configurations of cardinal 10 ? The question is difficult and seems not to be answered so far.

Unfortunately we will not contribute to this problem. We will give some elementary facts which will serve us as background for the next section about biquadratic forms.

Proposition 7.1. Let $\Omega \in \mathbb{P}^{2}$.

a) If $f$ is a positive semi-definite sextic such that $|\mathcal{Z}(f)|=10$, then $\mathcal{Z}(f)$ is a maximal configuration.

b) If $|\Omega|=8$, then $\Omega$ is not a maximal configuration.

c) Let $D_{1}, D_{2}, D_{3}$ (resp. $\Delta_{1}, \Delta_{2}, \Delta_{3}$ ) be the three lines of equations $x=$ $-z, x=0, x=z$ (resp. $y=-z, y=0, y=z)$. Let $\Omega=\cup_{i, j}\left(D_{i} \cap \Delta_{j}\right)$. Then, $\Omega$ is a 9-points maximal configuration.

d) The maximal sets containing $\Omega=\mathcal{Z}(R) \backslash\{(1,1,0),(1,-1,0)\}$ are exactly the sets $\mathcal{Z}(R)$ and $\Omega \cup\{P\}$ where $P$ is any point in $\mathbb{P}^{2}$ such that $P \notin$ $\{(1,1,0),(1,-1,0)\}$.

Proof. $\quad$ a) It is a direct consequence of the equality $B_{3,6}=10$.

b) Let $|\Omega|=8$. Let us consider two linearly independent cubics curves $p_{1}=0$ and $p_{2}=0$ going through all of these 8 points. Then, $p=p_{1}^{2}+p_{2}^{2}$ is a sextic such that $|\mathcal{Z}(p)|=9$.

c),d) Let $f$ be a positive semi-definite a sextic. We consider the coefficients of $f$ as variables. Each zero of $f$ is a singular point, hence we get 3 linearly independent equations. We then reduce the rows of our big linear system by using the Gauss algorithm and we are able to conclude our proofs. Completely similar techniques will be given with more details in the next section.

Of course, not all 9-points sets can be contained in a maximal configuration ; for instance take the case where the 9 points are lying in the same line. 
7.2. Biquadratic forms in $\mathcal{B} Q_{2,2}$. Let us recall that $\mathrm{BB}_{2,2}=2$. We have

Proposition 7.2. The maximal configurations in $\mathbb{P}^{1} \times \mathbb{P}^{1}$ are the sets $\Omega$ such that $|\Omega|=\left|\Pi_{1}(\Omega)\right|=\left|\Pi_{2}(\Omega)\right|=2$.

Proof. If $|\Omega| \neq 2$, then $\Omega$ is clearly not a maximal configuration.

Let us assume now that $|\Omega|=2$.

If $\left|\Pi_{1}(\Omega)\right|=1$, then up to linear change of coordinates, we may assume that $\Omega=\{(1,0 ; 1,0),(1,0 ; a, 1)\}$, with $a \in \mathbb{R}$. A biquadratic form $F$ vanishing at $\Omega$ will be a sum of squares of elements of the form $\alpha y s+\beta y t$ with $(\alpha, \beta) \in \mathbb{R}^{2}$. Hence, $|\mathcal{Z}(F)|=\infty$, namely $\Omega$ is not maximal. We have shown that the conditions are necessary.

Let us show now that they are sufficient. If $\left|\Pi_{1}(\Omega)\right|=2=\left|\Pi_{2}(\Omega)\right|$, then up to linear change of coordinates, we may assume that $\Omega=\{(1,0 ; 1,0),(0,1 ; 0,1)\}$. The biquadratic form $F=(x t)^{2}+(y s)^{2}$ is an example where $\mathcal{Z}(F)=\Omega$. Hence $\Omega$ is maximal.

We know from the identity $\mathrm{BB}_{2,2}=2$ that if the biquadratic form $F \in \mathcal{P} Q_{2,2}$ has 3 zeros or more, then (it is a sum of squares and) it has an infinite number of zeros. One may give a little bit more precise and geometric statement :

Proposition 7.3. Assume that $F$ vanishes at 3 different points $A_{1}, A_{2}, A_{3}$. Then, $F$ vanishes at any point $A_{4}$ satisfying the following identity on the cross ratios over $\mathbb{P}^{1}$ :

$$
\left[\Pi_{1}\left(A_{1}\right), \Pi_{1}\left(A_{2}\right), \Pi_{1}\left(A_{3}\right), \Pi_{1}\left(A_{4}\right)\right]=\left[\Pi_{2}\left(A_{1}\right), \Pi_{2}\left(A_{2}\right), \Pi_{2}\left(A_{3}\right), \Pi_{2}\left(A_{4}\right)\right] .
$$

Proof. Up to a linear change of coordinates, we may assume that $A_{1}=(1,0 ; 1,0)$, $A_{2}=(0,1 ; 0,1)$ and $A_{3}=\left(1, y_{0} ; 1, t_{0}\right)$. Then, $F$ is a sum of squares of bilinear forms of the type $\alpha x t+\beta y s$ where $(\alpha, \beta) \in \mathbb{R}^{2}$ is satisfying $\alpha t_{0}+\beta y_{0}=0$. Hence $F$ vanishes at $\left(1, y_{1} ; 1, t_{1}\right)$, for all $\left(y_{1}, t_{1}\right)$ such that $y_{0} t_{1}-y_{1} t_{0}=0$.

It remains to translate this last equality using the cross ratios over $\mathbb{P}^{1}$.

\subsection{Biquadratic forms in $\mathcal{B} Q_{3,2}$.}

Proposition 7.4. The maximal configurations in $\mathbb{P}^{2} \times \mathbb{P}^{1}$ are the sets $\Omega$ such that $|\Omega|=\left|\Pi_{1}(\Omega)\right|=\left|\Pi_{2}(\Omega)\right|=3$ and such that the 3 points in $\Pi_{1}(\Omega) \in \mathbb{P}^{2}$ are not lying on the same line.

Proof. If $|\Omega| \neq 3$, then $\Omega$ is clearly not a maximal configuration. Likewise, if $\left|\Pi_{1}(\Omega)\right| \neq 3$ or $\left|\Pi_{2}(\Omega)\right| \neq 3$.

Let us assume from now on that $|\Omega|=\left|\Pi_{1}(\Omega)\right|=\left|\Pi_{2}(\Omega)\right|=3$. Let $\Omega=$ $\left\{P_{1}, P_{2}, P_{3}\right\} \subset \mathcal{Z}(F)$ where $F \in \mathcal{P} Q(3,2)$.

Let us check that if $\Pi_{1}\left(P_{1}\right), \Pi_{1}\left(P_{2}\right), \Pi_{1}\left(P_{3}\right)$ are lying on the same line, then all the line is contained in $\Pi_{1}(\mathcal{Z}(F))$.

Up to linear change of coordinates, we may assume that

$$
A_{1}=(1,0,0 ; 1,0), A_{2}=(0,1,0 ; 0,1), A_{3}=\left(1, y_{2}, 0 ; 1, t_{2}\right)
$$

where $y_{2} t_{2} \neq 0$. Let us write $F=\sum_{i}\left(F_{i}\right)^{2}$, where

$$
F_{i}=a x s+b x t+c y s+d y t+e z s+f z t
$$

with $(a, b, c, d, e, f) \in \mathbb{R}^{6}$. By assumption, we have $a=d=0$ and $b t_{0}+c y_{0}=0$. Hence

$$
F_{i}=\lambda\left(y_{0} x t-t_{0} y s\right)+z(e s+f t)
$$


Thus, all the $F_{i}$ 's vanish at $\left(1, \beta, 0 ; t_{0}, \beta\right)$ for any $\beta \in \mathbb{R}$. Hence $\mathcal{Z}(F)$ is infinite and $\Omega$ is not maximal. We have shown that the condition of the proposition is necessary.

Let us now show it is sufficient. Up to a linear change of coordinates, we may assume that

$$
\Omega=\{(1,0,-1 ; 1,0),(0,1,-1 ; 0,1),(0,0,1 ;-\beta, \alpha)\}
$$

where $(\alpha, \beta) \in \mathbb{R}^{2} \backslash\{(0,0)\}$. We just consider the biquadratic form

$$
F=(x t)^{2}+(y s)^{2}+((x+y+z)(\alpha s+\beta t))^{2} \in \mathcal{P} Q_{3,2}
$$

and check that $\mathcal{Z}(F)=\Omega$.

Here is a statement on how to construct an infinite family of zeros when at least 4 zeros are given :

Proposition 7.5. Assume that $F$ vanishes at the 4 different points

$A_{1}=(1,0,0 ; 1,0), A_{2}=(0,1,0 ; 0,1), A_{3}=\left(x_{3}, y_{3}, z_{3} ; 1, t_{3}\right), A_{4}=\left(x_{4}, y_{4}, z_{4} ; 1, t_{4}\right)$.

Then, $F$ vanishes at any point $A_{5}=\left(x_{5}, y_{5}, z_{5} ; 1, t_{5}\right)$ satisfying :

$$
\operatorname{det}\left(\begin{array}{ccc}
z_{3} & t_{3} & y_{3} \\
z_{4} & t_{4} & y_{4} \\
z_{5} & t_{5} & y_{5}
\end{array}\right)=\operatorname{det}\left(\begin{array}{ccc}
t_{3} z_{3} & t_{3} & y_{3} \\
t_{4} z_{4} & t_{4} & y_{4} \\
t_{5} z_{5} & t_{5} & y_{5}
\end{array}\right)=0
$$

Proof. Let $F \in \mathcal{P} Q_{3,2}$. By Proposition 2.2, each point $A_{1}, A_{2}, A_{3}, A_{4}$ is a singular point of the biquadratic form $F$, and hence it yields 4 linear equations whose variables are the coefficients of $F$. Namely, let us write

$$
F=s^{2}\left(a_{0} x^{2}+a_{1} x y+a_{3} y^{2}+a_{2} x z+a_{4} y z+a_{5} z^{2}\right)
$$

$$
\begin{aligned}
& +2 s t\left(b_{0} x^{2}+b_{1} x y+b_{3} y^{2}+b_{2} x z+b_{4} y z+b_{5} z^{2}\right) \\
& +t^{2}\left(c_{0} x^{2}+c_{1} x y+c_{3} y^{2}+c_{2} x z+c_{4} y z+c_{5} z^{2}\right)
\end{aligned}
$$

Since $A_{1}$ and $A_{2}$ are critical points, we have

$$
a_{0}=a_{1}=a_{2}=b_{0}=b_{3}=c_{1}=c_{3}=c_{4}=0 .
$$

With respect to the remaining variables

$$
X=\left(a_{3}, a_{4}, a_{5}, b_{1}, b_{2}, b_{4}, b_{5}, c_{0}, c_{2}, c_{5}\right),
$$

the fact that $A_{3}$ and $A_{4}$ are critical points yields the equations

$$
A X^{T}=0
$$

where

$$
A=\left(\begin{array}{l}
A_{3} \\
A_{4}
\end{array}\right)
$$

and

$$
A_{k}=\left(\begin{array}{cccccccccc}
t_{k}^{2} & 2 t_{k} y_{k} & y_{k}^{2} & 2 t_{k} z_{k} & t_{k}^{2} z_{k} & y_{k} z_{k} & 2 t_{k} y_{k} z_{k} & z_{k}^{2} & 2 t_{k} z_{k}^{2} & t_{k}^{2} z_{k}^{2} \\
0 & 0 & 0 & 2 t_{k} & t_{k}^{2} & y_{k} & 2 t_{k} y_{k} & 2 z_{k} & 4 t_{k} z_{k} & 2 t_{k}^{2} z_{k} \\
t_{k} & y_{k} & 0 & z_{k} & t_{k} z_{k} & 0 & y_{k} z_{k} & 0 & z_{k}^{2} & t_{k} z_{k}^{2} \\
0 & 2 t_{k} & 2 y_{k} & 0 & 0 & z_{k} & 2 t_{k} z_{k} & 0 & 0 & 0
\end{array}\right) .
$$


First, let us assume we are in the generic case, namely $z_{3} y_{4}-z_{4} y_{3} \neq 0$. We reduce the rows of our matrix $A$ with the Gauss algorithm to a matrix $\widetilde{A}=\left(\operatorname{Id}_{7} \mid B\right)$ where $\operatorname{Id}_{7} \in \mathbb{R}^{8 \times 8}$ is the identity matrix and $B$ is the matrix :

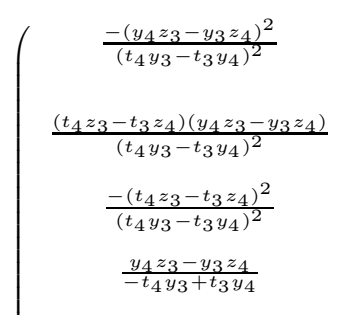

0

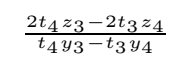

0

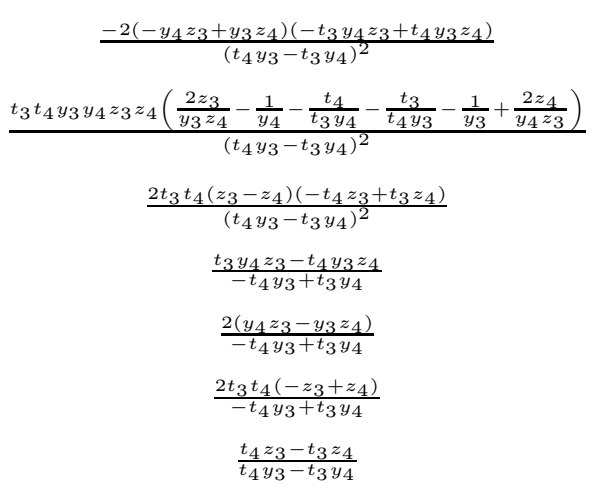

0

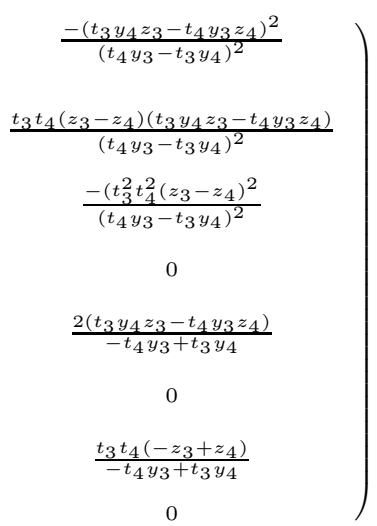

0

Next, we check, under the conditions (17), that the equation $F\left(A_{5}\right)=0$ corresponds to a row which is a linear combination of the rows of $A$. Indeed, a computation shows that the row reduction of the matrix $A$ augmented with the new row given by $F\left(A_{5}\right)=0$ is the matrix $\widetilde{A}$ augmented with the trivial compatibility equation $0=0$.

In the non generic case, we proceed likewise, replacing $z_{4}$ with $z_{3} y_{4} / y_{3}$ in the matrix $A$.

7.4. Biquadratic forms in $\mathcal{B} Q_{3,3}$. Contrarily to what we have done in the previous subsections, we are not able yet to describe all the possible maximal configurations in $\mathbb{P}^{2} \times \mathbb{P}^{2}$. We first give elementary negative results :

Proposition 7.6. Let $\Omega \in \mathbb{P}^{2} \times \mathbb{P}^{2}$ be such that $|\Omega| \geq 4$.

a) If there are $P_{1}, P_{2} \in \Omega$ such that $\Pi_{1}\left(P_{1}\right)=\Pi_{1}\left(P_{2}\right)$, then $\Omega$ is not maximal.

b) If $\Pi_{1}(\Omega)$ contains 4 points lying on the same line, then $\Omega$ is not maximal.

c) If $\Omega$ contains 3 points $P_{1}, P_{2}, P_{3}$ such that $\Pi_{1}\left(P_{1}\right), \Pi_{1}\left(P_{2}\right), \Pi_{1}\left(P_{3}\right)$ are lying on the same line and $\Pi_{2}\left(P_{1}\right), \Pi_{2}\left(P_{2}\right), \Pi_{2}\left(P_{3}\right)$ are lying on the same line, then $\Omega$ is not maximal.

d) If $\Omega$ is maximal, then we may assume, up to a linear change of coordinates, that it contains the points $(1,0,0 ; 1,0,0),(0,1,0 ; 0,1,0),(0,0,1 ; 0,0,1)$.

Proof. $\quad$ a) It is a direct application of Lemma 5.2.

b) Let $\left\{P_{1}, P_{2}, P_{3}, P_{4}\right\} \subset \Omega$ be such that $\Pi_{1}\left(P_{1}\right), \Pi_{1}\left(P_{2}\right), \Pi_{1}\left(P_{3}\right), \Pi_{1}\left(P_{4}\right)$ are lying on the line of equation $z=0$. We may assume that

$$
\begin{array}{ll}
P_{1}=(1,0,0 ; 1,0,0), & P_{2}=(0,1,0 ; 0,1,0), \\
\Pi_{1}\left(P_{3}\right)=\left(x_{1}, 1,0\right), & \Pi_{1}\left(P_{4}\right)=\left(x_{2}, 1,0\right) .
\end{array}
$$

Let $F \in \mathcal{P} Q_{3,3}$ be such that $\Omega \subset \mathcal{Z}(F)$. We set $G=F(x, y, 0 ; s, t, u)$. Then, we have $G \in \mathcal{P} Q_{2,3}$ and $|\mathcal{Z}(G)|>3$. Hence, $|\mathcal{Z}(G)|=\infty$ and also $|\mathcal{Z}(F)|=\infty$, which shows that $\Omega$ is not maximal.

c) As in the previous case, we may assume that

$$
P_{1}=(1,0,0 ; 1,0,0), P_{2}=(0,1,0 ; 0,1,0), P_{3}=\left(x_{0}, 1,0 ; s_{0}, 1,0\right)
$$

with $x_{0} s_{0} \neq 0$. 
Let $F \in \mathcal{P} Q_{3,3}$ be such that $\Omega \subset \mathcal{Z}(F)$. Let $G=F(x, y, 0 ; s, t, 0)$. We have $G \in \mathcal{P} Q_{2,2}$ and $|\mathcal{Z}(G)|>2$. Hence, $|\mathcal{Z}(G)|=\infty$ and also $|\mathcal{Z}(F)|=\infty$, which shows that $\Omega$ is not maximal.

d) We just repetitively use the previous case.

Next, we give one example of maximal configuration, namely the Choi set $\mathcal{C}$ as defined in (4). Again our method relies on basic linear algebra.

Proposition 7.7. The Choi set $\mathcal{C}$ is maximal in $\mathbb{P}^{2} \times \mathbb{P}^{2}$.

Proof. Let $F \in \mathcal{P} Q_{3,3}$ be such that $\mathcal{Z}(F)$ contains the whole set $\mathcal{C}$ and an extra point $B_{4}=\left(x_{4}, y_{4}, z_{4}, s_{4}, t_{4}, u_{4}\right)$. According to Proposition 2.2, each point of $\mathcal{Z}(F)$ is a singular point of the biquadratic form $F$, and hence yields 5 independent linear equations whose variables are the coefficients of $F$. Namely, let us write

$$
\begin{aligned}
F= & s^{2}\left(a_{0} x^{2}+a_{1} x y+a_{2} x z+a_{3} y^{2}+a_{4} y z+a_{5} z^{2}\right) \\
& +2 s t\left(b_{0} x^{2}+b_{1} x y+b_{2} x z+b_{3} y^{2}+b_{4} y z+b_{5} z^{2}\right) \\
& +2 s u\left(c_{0} x^{2}+c_{1} x y+c_{2} x z+c_{3} y^{2}+c_{4} y z+c_{5} z^{2}\right) \\
& +t^{2}\left(d_{0} x^{2}+d_{1} x y+d_{2} x z+d_{3} y^{2}+d_{4} y z+d_{5} z^{2}\right) \\
& +2 t u\left(e_{0} x^{2}+e_{1} x y+e_{2} x z+e_{3} y^{2}+e_{4} y z+e_{5} z^{2}\right) \\
& +u^{2}\left(f_{0} x^{2}+f_{1} x y+f_{2} x z+f_{3} y^{2}+f_{4} y z+f_{5} z^{2}\right) .
\end{aligned}
$$

In the generic case (i.e. when $s_{4} y_{4}-t_{4} z_{4} \neq 0$ ), the line reduction with the Gauss algorithm gives :

$$
\left\{\begin{array}{l}
a_{0}=a_{1}=a_{2}=a_{4}=a_{5}=0, \\
b_{0}=b_{1}=b_{2}=b_{3}=b_{5}=0, \\
c_{0}=c_{2}=c_{3}=c_{4}=c_{5}=0, \\
d_{0}=d_{1}=d_{2}=d_{3}=d_{4}=0, \\
e_{0}=e_{1}=e_{3}=e_{4}=e_{5}=0, \\
f_{1}=f_{2}=f_{3}=f_{4}=f_{5}=0, \\
a_{3}=\frac{f_{0}\left(u_{4} x_{4}-t_{4} z_{4}\right)^{2}}{\left(s_{4} y_{4}-t_{4} z_{4}\right)^{2}}, \\
b_{4}=-\frac{f_{0}\left(u_{4} x_{4}-s_{4} y_{4}\right)\left(u_{4} x_{4}-t_{4} z_{4}\right)}{\left(s_{4} y_{4}-t_{4} z_{4}\right)^{2}}, \\
c_{1}=-\frac{f_{0}\left(u_{4} x_{4}-t_{4} z_{4}\right)}{\left(s_{4} y_{4}-t_{4} z_{4}\right)}, \\
d_{5}=\frac{f_{0}\left(u_{4} x_{4}-s_{4} y_{4}\right)^{2}}{\left(s_{4} y_{4}-t_{4} z_{4}\right)^{2}}, \\
e_{2}=\frac{f_{0}\left(u_{4} x_{4}-s_{4} y_{4}\right)}{s_{4} y_{4}-t_{4} z_{4}} .
\end{array}\right.
$$


These values of the variables correspond to the biquadratic form :

$$
F=f_{0}\left(\frac{\left(s_{4} y_{4}-t_{4} z_{4}\right) u x+s y\left(-u_{4} x_{4}+t_{4} z_{4}\right)+\left(u_{4} x_{4}-s_{4} y_{4}\right) t z}{s_{4} y_{4}-t_{4} z_{4}}\right)^{2} .
$$

Since it is a square having more than 6 zeros, it necessarily has an infinite number of zeros. Then, $\mathcal{C} \cup\left\{B_{4}\right\}$ is not contained in a maximal set.

To end, we have to consider the non-generic case, namely when $s_{4} y_{4}-t_{4} z_{4}=0$.

If, $y_{4} \neq 0$, then by line reducing the system, we get $F=d_{5}(s y-t z)^{2}$. Likewise, if $z_{4} \neq 0$.

We have shown that $\mathcal{C}$ is maximal.

One idea to show that $\mathrm{BB}_{3,3}=7$ (if it were true !) would be to consider $F \in \mathcal{B} Q_{3,3}$ having 8 zeros, the coefficients of $F$ being viewed as 36 variables. The cancellation of the partial derivatives at the first three zeros which are assumed to be $(1,0,0 ; 1,0,0),(0,1,0 ; 0,1,0),(0,0,1 ; 0,0,1)$ shows that 15 variables are zeros and it remains 21 . The cancellation of the partial derivatives at the other five zeros (which are written with formal parameters) gives 25 equations. And we would like to construct then an infinite family of points $\left(P_{\lambda}\right)_{\lambda \in \mathbb{R}}$ such that the equation $F\left(P_{\lambda}\right)=0$ would be a linear combination of our previous 25 equations.

This method has been successful in the previous section for reproving that $\mathrm{BB}_{3,2}=3$ (Proposition 7.5) but here the computation becomes two expensive.

In relation with zeros configuration, one may also ask the following problem.

Question 7.8. Let $\Omega$ be a finite set in $\mathbb{P}^{2} \times \mathbb{P}^{2}$. Can we determine $\mathcal{P} Q(\Omega)$ the set of all positive semi-definite biquadratic forms $F \in \mathcal{P} Q_{3,3}$ such that $\Omega \subset \mathcal{Z}(F)$ ?

One main interest would be to find $\mathcal{P} Q(\Omega)$ when $\Omega$ is a maximal configuration. We are able to answer the question in the case of the Choi Set :

Theorem 7.9. With the notation of (马), all the biquadratic forms $F \in \mathcal{P} Q_{3,3}$ which are vanishing on the Choi Set $\mathcal{C}$ are described by

$$
M_{2}(F)=\left(\begin{array}{ccc}
\left(d_{5}+2 e_{2}+f_{0}+f_{3}\right) y^{2}+f_{3} z^{2} & \left(-d_{5}-e_{2}-f_{3}\right) y z & \left(-e_{2}-f_{0}-f_{3}\right) x y \\
\left(-d_{5}-e_{2}-f_{3}\right) y z & f_{3} x^{2}+d_{5} z^{2} & e_{2} x z \\
\left(-e_{2}-f_{0}-f_{3}\right) x y & e_{2} x z & f_{0} x^{2}+f_{3} y^{2}
\end{array}\right)
$$

with the conditions

$$
\left\{\begin{array}{l}
f_{0}=1 \\
1 \geq f_{3} \geq 0 \\
d_{5} \geq 0 \\
d_{5}-e_{2}^{2} \geq 0 \\
-e_{2}^{2}+d_{5}-d_{5} f_{3}-2 e_{2} f_{3}-f_{3}^{2} \geq 0 \\
-e_{2}^{2}+d_{5}-2 e_{2} f_{3}-f_{3}-f_{3}^{2} \geq 0
\end{array}\right.
$$

or $d_{5}=e_{2}=f_{0}=f_{3}=0$.

Proof. Let $F \in \mathcal{P} Q_{3,3}$ be such that $\mathcal{C} \subset \mathcal{Z}(F)$. We write $F$ as in (9), and consider the coefficients as variables. Writing down the 35 equations given by the cancellation of all the partial derivatives at all points of $\mathcal{C}$ yields that all the possible biquadratic forms can be described by the free parameters $d_{5}, e_{2}, f_{0}, f_{3}$. More precisely, we exactly get the $F$ 's whose associated matrix is $M_{2}(F)$ as given in (10). 
This matrix $M_{2}(F)$ if positive semi-definite for all $x, y, z$ if and only if all its symmetric minors are positive semi-definite for all $x, y, z$. The non-negativity of all the $1 \times 1$ and $2 \times 2$ minors gives the following conditions :

$$
\left\{\begin{array}{l}
f_{0} \geq 0 \\
f_{3} \geq 0 \\
d_{5} \geq 0 \\
d_{5}+2 e_{2}+f_{0}+f_{3} \geq 0 \\
d_{5} f_{0}-e_{2}^{2} \geq 0 \\
-e_{2}^{2}+d_{5} f_{0}-d_{5} f_{3}-2 e_{2} f_{3}-f_{3}^{2} \geq 0 \\
-e_{2}^{2}+d_{5} f_{0}-2 e_{2} f_{3}-f_{0} f_{3}-f_{3}^{2} \geq 0 .
\end{array}\right.
$$

The non-negativity of the $3 \times 3$ minor is

$$
p(x, y, z)=\operatorname{det}\left(M_{2}(F(x, y, z)) \geq 0 .\right.
$$

We compute :

$$
\begin{aligned}
p(x, y, z)= & -f_{3}\left(e_{2}^{2}-d_{5} f_{0}+2 e_{2} f_{3}+f_{3} f_{0}+f_{3}^{2}\right) x^{4} y^{2}+f_{0} f_{3}^{2} x^{4} z^{2} \\
& +f_{3}^{2}\left(d_{5}+2 e_{2}+f_{0}+f_{3}\right) y^{4} x^{2}-f_{3}\left(e_{2}^{2}+2 e_{2} f_{3}+f_{3}^{2}-d_{5} f_{0}+d_{5} f_{3}\right) y^{4} z^{2} \\
& +f_{3}\left(-e_{2}^{2}+d_{5} f_{0}\right) z^{4} x^{2}+d_{5} f_{3}^{2} z^{4} y^{2} \\
& +f_{3}\left(3 e_{2}^{2}+2 e_{2} f_{3}-f_{0} f_{3}+f_{3}^{2}-3 d_{5} f_{0}+d_{5} f_{3}\right) x^{2} y^{2} z^{2} .
\end{aligned}
$$

Let us note that $p$ depends only on $x^{2}, y^{2}, z^{2}$, hence we will introduce the underlying cubic and use the following :

Lemma 7.10. Let $c(x, y, z)=0$ be the equation of a cubic going by the points $(1,0,0),(0,1,0),(0,0,1)$. The equation of the cubic curve has the form

$$
\left\{\begin{array}{l}
c(x, y, z)=\alpha_{1} x^{2} y+\alpha_{2} x^{2} z+\beta_{1} y^{2} x+\beta_{2} y^{2} z+\gamma_{1} z^{2} x+\gamma_{2} z^{2} y+\delta x y z \\
\left(\alpha_{1}, \alpha_{2}, \beta_{1}, \beta_{2}, \gamma_{1}, \gamma_{2}, \delta\right) \in \mathbb{R}^{7}
\end{array}\right.
$$

Let us assume that all the coefficients except $\delta$ are non-negative. If $c(x, y, z)$ goes by $(1,1,1)$ and is non-negative on the following affine semi-lines (in the plane $z=1)$ :

$$
(x=y \geq 0), \quad(x=1, y \geq 0) \quad(y=1, x \geq 0),
$$

then $c(x, y, z)$ is non-negative on all the positive orthant $x \geq 0, y \geq 0, z \geq 0$. Moreover, its coefficients are satisfying

$$
\left\{\begin{array}{l}
\alpha_{1}+\alpha_{2}+\beta_{1}+\beta_{2}+\gamma_{1}+\gamma_{2}+\delta=0 \\
\alpha_{1}+\alpha_{2}=\beta_{2}+\gamma_{2} \\
\beta_{1}+\beta_{2}=\alpha_{2}+\gamma_{1}
\end{array}\right.
$$

Proof. We check that the positivity conditions on the semi-lines

$$
(x=y \geq 0), \quad(x=1, y \geq 0) \quad(y=1, x \geq 0),
$$

are equivalent to the cancellation of all the partial derivatives at $(1,1,1)$, which in turn gives the equalities (13).

To get lighter notations we write, for some $(a, b, s, t) \in \mathbb{R}_{+}^{4}$ :

$c=a x^{2} y+b x y^{2}+(-a+s) x^{2} z+(-b+t) y^{2} z+(a-s+t) x z^{2}+(b+s-t) y z^{2}-(a+b+s+t) x y z$. 
Let us consider the family of affine lines $L_{\lambda}: y=1+\lambda(x-1)$ going by $(1,1)$. We set

$c(x, 1+\lambda(x-1), 1)=(x-1)^{2} c_{\lambda}(x)=(x-1)^{2}(\lambda(a+b \lambda) x-(-1+\lambda)(s+\lambda(b-t)))$.

We have to distinguish several cases :

If $\lambda<0$, then we check that $c_{\lambda}(x) \geq 0$ for any $0 \leq x \leq \frac{\lambda-1}{\lambda}$. Indeed, it suffices to see that

$$
c_{\lambda}(0)=(\lambda-1)(\lambda(t-b)-s) \geq 0
$$

and

$$
c_{\lambda}\left(\frac{\lambda-1}{\lambda}\right)=(\lambda-1)(\lambda t+a-s) \geq 0 .
$$

Likewise, if $0 \leq \lambda \leq 1$ we check that for all $x \geq 0$

$$
c_{\lambda}(x) \geq c_{\lambda}(0) \geq 0 \text {. }
$$

And if $\lambda \geq 1$ we check that for all $x \geq \frac{\lambda-1}{\lambda}$

$$
c_{\lambda}(x) \geq c_{\lambda}\left(\frac{\lambda-1}{\lambda}\right) \geq 0 .
$$

We have shown that all the restrictions of $c$ to the intersection of any lines going by $(1,1)$ and the positive orthant take non negative values.

The conditions (12) imply that the form $p(x, y, z)$ is associated (by the change of variables $X=x^{2}, Y=y^{2}, Z=z^{2}$ ) to a cubic satisfying the conditions of Lemma 7.10. Hence $p(x, y, z)$ is non-negative. Namely the non-negativity of the $1 \times 1$ and $2 \times 2$ minors implies the non-negativity of the determinant.

In order to get a more geometric description, we deshomogenize by setting $f_{0}=1$ (if $f_{0}=0$, then $M=0$ ) and we consider the Euclidean space with coordinates $\left(d_{5}, e_{2}, f_{3}\right)$. Looking at the system of inequalities (12), we note that if $f_{3} \geq 0$ and $f_{5} \geq 0$, then the sixth inequality $-e_{2}^{2}+d_{5}-d_{5} f_{3}-2 e_{2} f_{3}-f_{3}^{2} \geq 0$ implies that $1-f_{3} \geq 0$. There are two cases.

The first is $f_{3}<1$. Then, it gives $d_{5}+2 e_{2}+1+f_{3} \geq\left(e_{2}+1\right)^{2}$ and hence the fourth inequality of (12) is obviously satisfied.

The second case is $f_{3}=1$, then $e_{2}=-1$ and again the fourth inequality is obviously implied by the sixth.

Thus, system (12) is equivalent to system (11).

Remark 7.11. The set $\mathcal{P} Q(\mathcal{C})$ is obviously convex since its defining conditions are convex with respect to the coefficients of the biquadratic forms.

The example $M_{0}$ in subsection 6.3 .2 corresponds to $\left(d_{5}, e_{2}, f_{3}\right)=(1,-1,1)$, the only point which belongs to the three hypersurfaces

$$
\left\{\begin{array}{l}
d_{5}-e_{2}^{2}=0 \\
-e_{2}^{2}+d_{5}-d_{5} f_{3}-2 e_{2} f_{3}-f_{3}^{2}=0 \\
-e_{2}^{2}+d_{5}-2 e_{2} f_{3}-f_{3}-f_{3}^{2}=0
\end{array}\right.
$$

which are delimiting the boundary of $\mathcal{P} Q(\mathcal{C})$. 
Looking carefully at the proof of Lemma 7.10, we note that $\mathcal{Z}(F)$ will be strictly greater than $\mathcal{C}$ only if $(t, s)=(a, 0)$ or $(s, t)=(0, b)$ or $t-s+a=0$ or $t-s-b=0$. In each case, we get $f_{3}=0$.

Hence, Theorem 7.9 gives a family of positive semi-definite biquadratic forms with parameters $\left(d_{5}, e_{2}, f_{0}, f_{3}\right)$ which are not sum of squares until $f_{3}=0$. More precisely, the sum of squares in the family $\mathcal{P} Q(\mathcal{C})$ are points corresponding to $f_{3}=0$ and delimited by the parabola $d_{5} \geq e_{2}^{2}$. The associated homogenized sum of squares identity is

$$
F=\left(x u+e_{2} z t-\left(e_{2}+f_{0}\right) y s\right)^{2}+\left(d_{5} f_{0}-e_{2}^{2}\right)(z t-y s)^{2} .
$$

\section{Quadratic Determinantal REPRESEntations}

Historically, a determinantal representation for a form $p(x)$ of degree $k$ in the variables $x=\left(x_{0}, x_{1}, x_{2}\right)$ is an identity of the kind

$$
p(x)=\lambda \operatorname{det}\left(x_{0} A_{0}+x_{1} A_{1}+x_{2} A_{2}\right)
$$

where $\lambda \in \mathbb{R}_{+}$and $A_{0}, A_{1}, A_{2}$ are matrices in $\mathbb{R}^{k \times k}$. The study of determinantal representations of polynomials goes back to the end of 19 th century, when were studied the relation between the determinantal representations and the geometry of the curve defined by the form.

More recently, determinantal representations over the reals where considered (see $\mathrm{Vi}$ ), and of particular interest in applications such as in Control theory, is the /it symmetric and unitary determinantal representations when the $A_{i}$ 's are symmetric and $A_{0}$ is the identity matrix (see [HV]).

In this last section, we would like to introduce a discussion about what could happen if we replace the linear pencil with a quadratic one. Namely, we would like to deal with Positive semi-definite quadratic determinantal representations. The new problem would be to write a given form $p(x)$ of degree $2 k$ as

$$
p(x)=\lambda \operatorname{det}(M(x))
$$

with $\lambda \in \mathbb{R}_{+}$and

$$
M(x)=x_{0}^{2} M_{0}+x_{0} x_{1} M_{1}+x_{0} x_{2} M_{2}+x_{1}^{2} M_{3}+x_{2} x_{1} M_{4}+x_{2}^{2} M_{5}
$$

where the $M_{i}$ 's are symmetric matrices in $\mathbb{R}^{k \times k}$ and such that $M(x)$ is positivesemi-definite for all $x \in \mathbb{R}^{3}$.

Given a biquadratic form $F(x ; s) \in \mathcal{B} Q_{3,3}$ we have two canonical associated matrices $\Pi_{x}(F)$ and $\Pi_{s}(F)$ which are positive semi-definite if and only if $F$ is. Hence positive semi-definite quadratic determinantal representations are naturally related to positive semi-definite biquadratic forms.

But it seems not so easy to develop a general theory of positive semi-definite quadratic determinantal representations, the positivity condition being the most difficult to handle. Let us just discuss two examples.

Since $\mathrm{BB}(2,2)=2$, we know that the form

$$
p(x, y, z)=\left(x^{2}-z^{2}\right)^{2}+\left(y^{2}-z^{2}\right)^{2}
$$


cannot have a positive semi-definite quadratic determinantal representation, although without the positivity condition, we may write

$$
p(x, y, z)=-\operatorname{det}\left(\begin{array}{cc}
x^{2}-z^{2} & y^{2}-z^{2} \\
y^{2}-z^{2} & -x^{2}+z^{2}
\end{array}\right) .
$$

If $\mathrm{BB}_{3,3}=7$ (or at least if $\mathrm{BB}_{3,3}<10$ ) as conjectured in the previous section, then we would derive that the Robinson polynomial $R(x, y, z)$ (as defined in (5)) would not admit any positive semi-definite quadratic determinantal representation.

Whereas we know that the polynomial

$$
S(x, y, z)=y^{2} x^{4}+y^{4} z^{2}+x^{2} z^{4}-3 x^{2} y^{2} z^{2}
$$

admits such one (Proposition 6.6).

One may also ask the same question for the celebrated Motzkin polynomial

$$
M(x, y, z)=z^{6}+y^{2} x^{4}+y^{4} x^{2}-3 x^{2} y^{2} z^{2},
$$

which has 6 zeros in $\mathbb{P}^{2}$.

\section{REFERENCES}

[C1] Choi, M.D Positive Semidefinite Biquadratic Forms, Linear Algebra and Appl. 12 (1975), no. 2, 95-100.

[C2] Choi, M.D Completely Positive Linear Maps and Complex Matrices, Linear Algebra and Appl. 10 (1975), no. 3, 285-290.

[Ca] A.P. Calderon, A note on Biquadratic Forms, Linear Algebra Appl. 7 (1973), 175-177

[CLR1] M.D. Choi, T.Y. Lam, B. Reznick, Real zeros of positive semidefinite forms. I. Math. Z. 171 (1980), no. 1, 1-26.

[CLR2] M.D. Choi, T.Y. Lam, B. Reznick, Sums of squares of real polynomials., K-theory and algebraic geometry: connections with quadratic forms and division algebras (Santa Barbara, CA, 1992), 103-126, Proc. Sympos. Pure Math., 58, Part 2, Amer. Math. Soc., Providence, RI, 1995.

[EGH] D. Eisenbud, M. Green, J. Harris, Cayley-Bacharach theorems and conjectures, Bull. Amer. Math. Soc. (N.S.) 33 (1996), 295-324

[Ha] J. Harris. Algebraic Geometry. Springer GTM 133, 1992.

[Hi] Hilbert, D. Über die Darstellung definiter Formen als Summe von Formquadraten. Math. Ann. 32, 342-350 (1888)

[HV] J.W. Helton, V. Vinnikov, Linear Matrix Inequality Representation of Sets, Comm. Pure and Appl. Math. 60 (2007), no. 5, 654-674.

[LS] D.B. Leep, C. L. Starr, Estimates of the Pythagoras number of $\mathbb{R}_{m}\left[x_{1}, \ldots, x_{n}\right]$ through lattice points and polytopes. Discrete Math. 308 (2008), no. 23, 5771-5781.

[Pf] A. Pfister, On Hilbert's theorem about ternary quartics, Algebraic and Arithmetic Theory of Quadratic Forms, Contemp. Math. 344, AMS, Providence, RI, 2004, pp. 295 ?301.

[Qz] R. Quarez, Piecewise Certificates of Positivity for matrix polynomials, arXiv:1001.1277v1

[SZ] Ł. Skowronek and K. Życzkowski, Positive maps, positive polynomials and entanglement witnesses, J. Phys. A42, 325302 (2009).

[Te] F. J. Terpstra, Die Darstellung biquadratischer Formen als Summen yon Quadraten mit Anwendung auf die Variationsrechnung., Math. Ann. 116 (1939), no1, 166-180

[Vi] V. Vinnikov, Self-adjoint determinantal representations of real plane curves, Math. Ann. 296 (1993), 453-479

Ronan Quarez, IRMAR (CNRS, URA 305), Université de Rennes 1, Campus de Beaulieu, 35042 Rennes Cedex, France

E-mail address: e-mail : ronan.quarez@univ-rennes1.fr 